\title{
Change of Variable in Spaces of Mixed Smoothness and Numerical Integration of Multivariate Functions on the Unit Cube
}

\author{
Van Kien Nguyen ${ }^{1,2}$ - Mario Ullrich ${ }^{3}$ - Tino Ullrich ${ }^{4}$
}

Received: 5 November 2015 / Revised: 21 November 2016 / Accepted: 18 January 2017 /

Published online: 3 March 2017

(C) The Author(s) 2017. This article is published with open access at Springerlink.com

\begin{abstract}
In a recent article, two of the present authors studied Frolov's cubature formulae and their optimality in Besov-Triebel-Lizorkin spaces of functions with dominating mixed smoothness supported in the unit cube. In this paper, we give a general result that the asymptotic order of the minimal worst-case integration error is not affected by boundary conditions in the above mentioned spaces. In fact, we propose two tailored modifications of Frolov's cubature formulae suitable for functions supported on the cube (not in the cube) that yield the same order of convergence up to a constant. This constant involves the norms of a "change of variable" and a "pointwise multiplication" mapping, respectively, between the function spaces of interest. We complement, extend, and improve classical results on the boundedness of change of variable mappings in Besov-Sobolev spaces of mixed smoothness. The second modification, suitable for classes of periodic functions, is based on a pointwise multiplication and is therefore most likely more suitable for applications than the (traditional) "change of variable" approach. These new theoretical insights are expected
\end{abstract}

Communicated by Vladimir N. Temlyakov.

$凶 \quad$ Mario Ullrich

mario.ullrich@jku.at

Van Kien Nguyen

kien.nguyen@uni-jena.de; kiennv@utc.edu.vn

Tino Ullrich

tino.ullrich@hcm.uni-bonn.de

1 Friedrich-Schiller-University Jena, Ernst-Abbe-Platz 2, 07737 Jena, Germany

2 University of Transport and Communications, Dong Da, Hanoi, Vietnam

3 Johannes Kepler University, Altenberger Str. 69, 4040 Linz, Austria

4 Hausdorff-Center for Mathematics, Endenicher Allee 62, 53115 Bonn, Germany 
to be useful for the design of new (and robust) cubature rules for multivariate functions on the cube.

Keywords Numerical integration - Change of variable $\cdot$ Periodization · Besov-Triebel-Lizorkin spaces $\cdot$ Dominating mixed smoothness

Mathematics Subject Classification 68Q25 - 65Y20 • 41A25 - 41A63 • 42B35 • 47B38

\section{Introduction}

This paper deals with the optimal (in order) approximation of multivariate integrals $\int_{\Omega} f(x) \mathrm{d} x$ with (non-)periodic integrands via cubature formulae of type

$$
Q_{n}(f):=\sum_{i=1}^{n} \lambda_{i} f\left(x^{i}\right),
$$

where $X_{n}:=\left\{x^{1}, \ldots, x^{n}\right\} \subset \Omega \subset \mathbb{R}^{d}$ denotes the set of given integration nodes and $\left(\lambda_{1}, \ldots, \lambda_{n}\right)$ denotes the vector of integration weights. This research area developed into several directions and attracted a lot of interest in the past 50 years, starting with the seminal papers by Korobov [20], Hlawka [18], and Bakhvalov [2]. Afterwards many authors contributed to the construction and analysis of optimal cubature formulae for multivariate functions (with bounded mixed derivative), see, e.g., Frolov [12], Bykovskii [3], Temlyakov [33-39], Dubinin [7,8], Skriganov [31], Triebel [43], Hinrichs et al. [16,17], Markhasin [23], Novak and Woźniakowski [26], Krieg and Novak [21], Dick and Pillichshammer [6], Dũng and Ullrich [10], Goda et al. [14,15], and Ullrich [45], to mention just a few. More historical comments and further references can be found at the end of this introduction as well as in the recent survey paper [9, Sect. 8.8, 8.9]. Nevertheless, several fundamental problems still remain unsolved. For instance, the construction of optimal (and easy to generate) point sets $X_{n}$ and corresponding weights in (1.1) leading to optimal (in order) cubature rules for a broad variety of function classes with dominating mixed smoothness is still the subject of intense research.

The concept of dominating mixed smoothness does not only connect discrepancy theory and optimal numerical integration, see [9, Sect. 8.8], it also plays an important role in many real-world problems. Several applications are modeled in Sobolev spaces with dominating mixed smoothness $H_{\text {mix }}^{s}$, see for instance Yserentant's book [51] for regularity properties of solutions of the electronic Schrödinger equation. To model multivariate kink functions that often occur in mathematical finance, e.g., the pricing of a European call option, whose pay-off function possesses a kink at the strike price [13], smoothness classes built on $L_{p}$ are appropriate. Such a kink can achieve smoothness $s=2$ in case $p=1$. The error bounds and numerical experiments in $[17,46]$ show that the convergence rate of the worst-case error for several cubature rules are determined by this regularity. 
Frolov's construction [12] dates back to the 1970s and gives a simple construction of admissible lattices that perform optimally with respect to several function classes of functions supported in the unit cube with mixed smoothness properties [46]. This will be the starting point for the construction and analysis of cubature formulae for the numerical integration of functions with nontrivial boundary data in this paper.

\subsection{Contribution and Main Results}

The main goal of the present paper is to construct cubature formulae that

(I) perform optimally in the sense of the minimal worst-case error (1.8) with respect to (non-)periodic Sobolev-Besov-Triebel-Lizorkin spaces of dominating mixed smoothness $\mathbf{B}_{p, \theta}^{s}$ and $\mathbf{F}_{p, \theta}^{s}$ on the unit $d$-cube, and

(II) are simple, robust, easy to implement, and universal in the sense that the algorithm does not depend on the specific parameters of the spaces.

The following two approaches reduce Problem (I) to the problem of numerical integration of functions with homogeneous boundary, where we know that the Frolov cubature formulae perform optimally in various settings [46].

The first approach being proposed by Bykovskii [3] shows that numerical integration in spaces with bounded mixed derivative is asymptotically not "harder" than the integration of functions with homogeneous boundary. A change of variable yields

$$
\int_{[0,1]^{d}} f(x) \mathrm{d} x=\int_{[0,1]^{d}}\left|\operatorname{det} \psi^{\prime}(x)\right| f(\psi(x)) \mathrm{d} x
$$

for some differentiable $\psi:[0,1]^{d} \rightarrow[0,1]^{d}$. Afterwards we apply a cubature formula (1.1) for the right-hand integrand in (1.2). The main observation is the fact that this approach results in a modified cubature formula,

$$
Q_{n}^{\psi}(f):=Q_{n}\left(\left|\operatorname{det} \psi^{\prime}\right| \cdot f \circ \psi\right)=\sum_{i=1}^{n} \lambda_{i}\left|\operatorname{det} \psi^{\prime}\left(x^{i}\right)\right| f\left(\psi\left(x^{i}\right)\right)
$$

Arranging the kernel $\psi$ in a way such that the function $\left|\operatorname{det} \psi^{\prime}(x)\right| f(\psi(x))$ preserves mixed smoothness properties and is supported in $[0,1]^{d}$, one can now use the above mentioned (optimal) methods to approximate the integral. Thus, the remaining step in analyzing the method (1.3) is to prove the preservation of mixed smoothness properties under change of variable. A straightforward choice for $\psi$ is certainly given by assuming a tensor product structure. That is, e.g., given univariate functions $\psi: \mathbb{R} \rightarrow \mathbb{R}$ that are integrated $C^{k}(\mathbb{R})$ bump functions $\varphi$ supported in $[0,1]$ and, with a slight abuse of notation, define $\psi\left(x_{1}, \ldots, x_{d}\right):=\psi\left(x_{1}\right) \cdot \ldots \psi \psi\left(x_{d}\right)$. A natural and simple choice is given by the family of polynomials

$$
\psi_{k}(t)=\left\{\begin{aligned}
& \int_{0}^{t} \xi^{k}(1-\xi)^{k} \mathrm{~d} \xi / \int_{0}^{1} \xi^{k}(1-\xi)^{k} \mathrm{~d} \xi: t \in[0,1], \\
& 1: t>1, \\
& 0: t<0
\end{aligned}\right.
$$


$k \in \mathbb{N}$, and we define

$$
\psi_{k}(x)=\psi_{k}\left(x_{1}\right) \ldots \psi_{k}\left(x_{d}\right)
$$

A second approach, which can be applied for numerical integration of multivariate periodic functions, is based on pointwise multiplication with a certain function and does not change the cubature nodes. To the best of our knowledge, this method has not been used before. In contrast to the considerations above, we will now restrict to function spaces on the $d$-torus $\mathbb{T}^{d}$, see Sect. 3.2 below; i.e., we want to numerically integrate functions that are 1-periodic in every component. To this end, we use a sufficiently smooth function $\psi: \mathbb{R}^{d} \rightarrow[0, \infty)$ being compactly supported $(\operatorname{supp}(\psi)=\Omega)$ such that

$$
\sum_{\ell \in \mathbb{Z}^{d}} \psi(x+\ell)=1, \quad x \in \mathbb{R}^{d} .
$$

Clearly, we need $\Omega \supsetneq[0,1]^{d}$. Then for any 1-periodic integrable function in each component, it holds that

$$
\begin{aligned}
\int_{\mathbb{R}^{d}} \psi(x) f(x) \mathrm{d} x & =\sum_{\ell \in \mathbb{Z}^{d}} \int_{[0,1]^{d}} \psi(x+\ell) f(x+\ell) \mathrm{d} x \\
& =\int_{[0,1]^{d}} f(x) \sum_{\ell \in \mathbb{Z}^{d}} \psi(x+\ell) \mathrm{d} x=\int_{[0,1]^{d}} f(x) \mathrm{d} x .
\end{aligned}
$$

Starting with a cubature formula $Q_{n}$ of type (1.1) for functions with support in $\Omega$, we modify as follows:

$$
\widetilde{Q}_{n}^{\psi}:=Q_{n}(\psi f)=\sum_{i=1}^{n} \psi\left(x^{i}\right) \lambda_{i} f\left(\left\{x^{i}\right\}\right),
$$

where $\{x\}=x-\lfloor x\rfloor$ has to be understood component-wise.

Let us define the minimal worst-case error:

$$
\operatorname{Int}_{n}\left(\mathbf{F}_{d}\right):=\inf _{Q_{n}} e\left(Q_{n}, \mathbf{F}_{d}\right), \quad n \in \mathbb{N}
$$

with respect to a function class $\mathbf{F}_{d}$ of $d$-variate (continuous) functions, where the infimum is taken over all cubature formulae of the form (1.1) and

$$
e\left(Q_{n}, \mathbf{F}_{d}\right):=\sup _{\|f\|_{\mathbf{F}_{d}} \leq 1}\left|I(f)-Q_{n}(f)\right|, \quad n \in \mathbb{N} .
$$


Our approach to analyze the cubature formulae (1.3) in spaces with mixed smoothness $\mathbf{F}_{d}=\mathbf{A}_{p, \theta}^{s}$ is to establish the boundedness of the operator

$$
\begin{aligned}
T_{d}^{\psi}: \mathbf{A}_{p, \theta}^{s} & \rightarrow \mathbf{A}_{p, \theta}^{s} \\
f & \mapsto\left|\operatorname{det} \psi^{\prime}(x)\right| f(\psi(x))
\end{aligned}
$$

for suitably chosen $\psi(x)$, where $\mathbf{A} \in\{\mathbf{B}, \mathbf{F}\}$. An important special case of our main result in Theorem 4.1 below gives the desired result for the Besov spaces $\mathbf{B}_{p, \theta}^{s}$ of dominating mixed smoothness using the change of variable kernel (1.5).

Theorem 1.1 Let $\Omega=[0,1]^{d}, 1 \leq p \leq \infty, 0<\theta \leq \infty$, and $s>0$. Further, let $k>\lfloor s\rfloor+1(k>\lfloor s\rfloor+2$ if $p=1)$ and $\psi_{k}$ as in (1.5). Then,

(i) $T_{d}^{\psi_{k}}: \mathbf{B}_{p, \theta}^{s} \rightarrow \mathbf{B}_{p, \theta}^{s}$ is a bounded mapping, and

(ii) provided that $\mathbf{B}_{p, \theta}^{s} \subset C\left(\mathbb{R}^{d}\right)$, a corresponding modified cubature formula (1.3) on $\mathbf{B}_{p, \theta}^{s}$ does not perform asymptotically worse than (1.1) performs on $\stackrel{\circ}{\mathbf{B}}_{p, \theta}^{s}$; i.e.,

$$
e\left(Q_{n}^{\psi_{k}}, \mathbf{B}_{p, \theta}^{s}\right) \lesssim e\left(Q_{n}, \stackrel{\circ}{\mathbf{B}}_{p, \theta}^{s}\right), \quad n \in \mathbb{N}
$$

The more restrictive condition in the case $p=1$ is actually not necessary, see Remark 4.7, but a proof of this statement would elongate the paper by some pages, so we leave it out.

An analogous result for the Triebel-Lizorkin spaces of dominating mixed smoothness $\mathbf{F}_{p, \theta}^{s}$ follows from Theorem 4.2 below. Note that we have to exclude the end points $p=1, \infty$.

Theorem 1.2 Let $\Omega=[0,1]^{d}, 1<p<\infty, 1<\theta \leq \infty$, and $s>0$. Further, let $k>\lfloor s\rfloor+1$ and $\psi_{k}$ as in (1.5). Then,

(i) $T_{d}^{\psi_{k}}: \mathbf{F}_{p, \theta}^{s} \rightarrow \mathbf{F}_{p, \theta}^{s}$ is a bounded mapping, and

(ii) provided that $\mathbf{F}_{p, \theta}^{s} \subset C\left(\mathbb{R}^{d}\right)$, a corresponding modified cubature formula (1.3) on $\mathbf{F}_{p, \theta}^{s}$ does not perform asymptotically worse than (1.1) performs on $\stackrel{\circ}{\mathbf{F}}_{p, \theta}^{s}$; i.e.,

$$
e\left(Q_{n}^{\psi_{k}}, \mathbf{F}_{p, \theta}^{s}\right) \lesssim e\left(Q_{n}, \stackrel{\circ}{\mathbf{F}}_{p, \theta}^{s}\right), \quad n \in \mathbb{N}
$$

Theorems 1.1 and 1.2 represent significant progress compared to the results in the literature $[3,8,36,38]$. Let us emphasize that the condition on the polynomial degree $k$ of the change of variable kernel (1.4) (or, equivalently, the condition on the smoothness of the kernel) only depends on the smoothness of the function space which significantly improves on Temlyakov's condition (1.11) below, where for $p \rightarrow 1$ the polynomial degree $k$ tends to infinity or it had to be replaced by a $C^{\infty}$ function of type (1.12). Note that in contrast to the pointwise multiplication in Theorems 1.3, 1.4 below, a boundedness result for the spaces $\mathbf{F}_{1, \theta}^{s}$ is still not known, see Remark 4.3(ii) below. Referring to (II) above, the numerical implementation of polynomials with very high degree is connected with certain issues on stability and robustness. In addition, the 
$C^{\infty}$-kernel in (1.12) has some obvious disadvantages, too. It involves terms that get very small and others that get very large at the same time. From that point of view, one may be interested in as simple as possible change of variable kernels with the least (smoothness) requirements. The functions in (1.4) are polynomials and therefore good for such a purpose. Therefore, the results above are not just an extension of the results from $[3,8,36,38]$ to more general function spaces. They essentially improve on the below stated classical results for $\mathbf{W}_{p}^{s}$ and $\mathbf{B}_{p, \theta}^{s}$ what concerns the choice of the change of variable kernel, which is an essential ingredient for the resulting modified cubature formula.

As a direct consequence of Theorems 1.1 and 1.2, we obtain the relations

$$
\operatorname{Int}_{n}\left(\AA_{p, \theta}^{s}\right) \asymp \operatorname{Int}_{n}\left(\mathbf{A}_{p, \theta}^{s}\left(\mathbb{T}^{d}\right)\right) \asymp \operatorname{Int}_{n}\left(\mathbf{A}_{p, \theta}^{s}\left([0,1]^{d}\right)\right), \quad n \in \mathbb{N},
$$

in the range of parameters given in Theorems 1.1 and 1.2 whenever the classes $\mathbf{A}_{p, \theta}^{s}$ consist of continuous functions. Let us mention the important special case

$$
\operatorname{Int}_{n}\left(\stackrel{\circ}{\mathbf{W}}_{p}^{s}\right) \asymp \operatorname{Int}_{n}\left(\mathbf{W}_{p}^{s}\left(\mathbb{T}^{d}\right)\right) \asymp \operatorname{Int}_{n}\left(\mathbf{W}_{p}^{s}\left([0,1]^{d}\right)\right), \quad n \in \mathbb{N}
$$

for $1<p<\infty$ and $s>1 / p$ taking into account that $\mathbf{F}_{p, 2}^{s}=\mathbf{W}_{p}^{s}$. Note that this includes the case of small smoothness, which is present if $2<p<\infty$ and $1 / p<s \leq 1 / 2$, see $[35,46]$.

In contrast to (1.9), the remaining step for analyzing (1.7) on 1-periodic function spaces is to establish the boundedness of pointwise multiplier mappings,

$$
\begin{aligned}
\widetilde{T}_{d}^{\psi}: \mathbf{A}_{p, \theta}^{s}\left(\mathbb{T}^{d}\right) & \rightarrow \mathbf{A}_{p, \theta}^{s}\left(\mathbb{R}^{d}\right) \\
f(x) & \mapsto \psi(x) f(x) .
\end{aligned}
$$

There is a rich theory concerning pointwise multiplication for isotropic spaces $B_{p, \theta}^{s}\left(\mathbb{R}^{d}\right), F_{p, \theta}^{s}\left(\mathbb{R}^{d}\right)$, see Runst and Sickel [28]. To the best of our knowledge, the field is rather open for spaces of dominating mixed smoothness $\mathbf{A}_{p, \theta}^{s}$. In contrast to the theorems above on change of variable, we are able to include the quasi-Banach case $(\min \{p, \theta\}<1)$ within this framework. This is why the natural lower smoothness restrictions enter, namely $s>\sigma_{p}$ and $s>\sigma_{p, \theta}$, respectively, where

$$
\sigma_{p}:=\max \left\{\frac{1}{p}-1,0\right\} \text { and } \sigma_{p, \theta}:=\max \left\{\frac{1}{p}-1, \frac{1}{\theta}-1,0\right\} \text {. }
$$

For the B-spaces, the result looks as follows:

Theorem 1.3 Let $0<p, \theta \leq \infty, s>\sigma_{p}$, and $\psi \in C_{0}^{k}(\Omega)$ with $k \geq\lfloor s\rfloor+1$. Then,

(i) $\widetilde{T}_{d}^{\psi}: \mathbf{B}_{p, \theta}^{s}\left(\mathbb{T}^{d}\right) \rightarrow \mathbf{B}_{p, \theta}^{s}$ is a bounded mapping, and

(ii) provided that $\mathbf{B}_{p, \theta}^{s} \subset C\left(\mathbb{R}^{d}\right)$ and $\psi$ satisfies (1.6), a corresponding modified cubature formula (1.7) on $\mathbf{B}_{p, \theta}^{s}\left(\mathbb{T}^{d}\right)$ does not perform asymptotically worse than (1.1) performs on $\stackrel{\circ}{\mathbf{B}}_{p, \theta}^{s}(\Omega)$; i.e., 


$$
e\left(\widetilde{Q}_{n}^{\psi}, \mathbf{B}_{p, \theta}^{s}\left(\mathbb{T}^{d}\right)\right) \lesssim e\left(Q_{n}, \stackrel{\circ}{B}_{p, \theta}^{s}(\Omega)\right), \quad n \in \mathbb{N}
$$

The result for the $\mathbf{F}$-spaces is similar, but we have to exclude the case $p=\infty$.

Theorem 1.4 Let $0<p<\infty, 0<\theta \leq \infty, s>\sigma_{p, \theta}$, and $\psi \in C_{0}^{k}(\Omega)$ with $k \geq\lfloor s\rfloor+1$. Then,

(i) $\widetilde{T}_{d}^{\psi}: \mathbf{F}_{p, \theta}^{s}\left(\mathbb{T}^{d}\right) \rightarrow \mathbf{F}_{p, \theta}^{s}$ is a bounded mapping, and

(ii) provided that $\mathbf{F}_{p, \theta}^{s} \subset C\left(\mathbb{R}^{d}\right)$ and $\psi$ satisfies (1.6), a corresponding modified cubature formula (1.7) on $\mathbf{F}_{p, \theta}^{s}\left(\mathbb{T}^{d}\right)$ does not perform asymptotically worse than (1.1) performs on $\stackrel{\circ}{\mathbf{F}}_{p, \theta}^{s}(\Omega)$; i.e.,

$$
e\left(\widetilde{Q}_{n}^{\psi}, \mathbf{F}_{p, \theta}^{s}\left(\mathbb{T}^{d}\right)\right) \lesssim e\left(Q_{n}, \stackrel{\circ}{\mathbf{F}}_{p, \theta}^{s}(\Omega)\right), \quad n \in \mathbb{N}
$$

Both theorems immediately imply the two-sided relation

$$
\operatorname{Int}_{n}\left(\AA_{p, \theta}^{s}\right) \asymp \operatorname{Int}_{n}\left(\mathbf{A}_{p, \theta}^{s}\left(\mathbb{T}^{d}\right)\right), \quad n \in \mathbb{N},
$$

in the above range of parameters if additionally $\mathbf{A}_{p, \theta}^{s} \hookrightarrow C\left(\mathbb{R}^{d}\right)$ holds true.

\subsection{State of the Art and Relevant Literature}

Numerical integration in periodic Besov spaces of dominating mixed smoothness $\mathbf{B}_{p, \theta}^{s}$ has been studied in $[7,8,10,17,39]$, see also Section 8 in the recent survey [9]. There are many results for $\mathbf{W}_{p}^{s}\left(\mathbb{T}^{d}\right)$ and $\mathbf{B}_{p, \infty}^{s}\left(\mathbb{T}^{d}\right)$ in this direction, see for instance [38] and the references therein. The recent contribution [17] especially deals with periodic Besov spaces of dominating mixed smoothness and analyzes cubature formulae on digital nets. Except for the Fibonacci cubature rule [10,35-38] in $d=2$, the discussed methods in $[17,43]$ are either not optimal or the optimal methods only work for specific (restricted) sets of parameters, for instance small smoothness $s<2$ in [17]. In other words, the cubature formula is optimal for low smoothness but is not able to benefit from higher regularity. Recently, the authors in [14,15] proposed new Quasi-Monte Carlo methods for $\mathbf{W}_{2}^{s}\left([0,1]^{d}\right)$ that are optimal in order for all $s>1 / 2$ but heavily depend on the smoothness parameter $s$. One goal of this paper was to get rid (or at least weaken) such dependencies.

To gain this, we use a reduction of the problem to the $\AA_{p, \theta}^{s}$ setting via tailored transformations in connection with the Frolov cubature formulae $[12,36]$, which recently attracted significant interest [19,21,44-46]. It has been already proved by Bykovskii [3] that $T_{d}^{\psi_{k}}$ is bounded in the Sobolev space with dominating mixed smoothness $\mathbf{W}_{2}^{s}$ for $s \in \mathbb{N}$ if $k \geq 2 s+1$. This result has been extended by Temlyakov, see [36, Thm. IV.4.1], to Sobolev spaces $\mathbf{W}_{p}^{s}, s \in \mathbb{N}, 1<p<\infty$, under the condition

$$
k \geq\left\lfloor\frac{s p}{p-1}\right\rfloor+1
$$


Under the same condition, Temlyakov [36, Lem. IV.4.9] showed the boundedness of $T_{d}^{\psi_{k}}: \mathbf{B}_{p, \infty}^{s} \rightarrow \mathbf{B}_{p, \infty}^{s}$ in the Hölder-Nikol'skij spaces if $1<p \leq \infty$ and $s>1$.

Using a $C^{\infty}(\mathbb{R})$-kernel instead of (1.4), namely

$$
\psi(t)=\left\{\begin{aligned}
\int_{0}^{t} e^{-\frac{1}{\xi(1-\xi)} \mathrm{d} \xi / \int_{0}^{1} e^{-\frac{1}{\xi(1-\xi)}} \mathrm{d} \xi}: t \in[0,1], \\
1: t>1, \\
0: t<0
\end{aligned}\right.
$$

Dubinin [7,8] proved boundedness for the Besov spaces $\mathbf{B}_{p, \theta}^{s}$ if $1 \leq p, \theta \leq \infty$, and $s>1 / p$. Temlyakov [38] considered the spaces $\mathbf{W}_{p}^{s}, s \in \mathbb{N}, 1 \leq p \leq \infty$. With this particular choice, both authors were able to incorporate the important case $p=1$ (see the discussion above) in the $\mathbf{B}$ and $\mathbf{W}$ framework. Note that no boundedness result is known for the $\mathbf{F}_{1, \theta}^{s}$ spaces, see Remark 4.3(ii) below.

From a tractability point of view, i.e., if one is interested in the dependence of the involved constants on the dimension, every method of this type will most likely be intractable. Some results on this issue in the Hilbert space setting can be found in Kuo et al. [22], see also Nuyens and Cools [27] for numerical experiments.

\subsection{Organization of the Paper}

The paper is organized as follows. In Sect. 2, we collect the required tools from Fourier analysis, especially maximal inequalities. Section 3 is devoted to the definition and properties of the function spaces under consideration. There we characterize BesovTriebel-Lizorkin spaces by mixed iterated differences that turn out to be the crucial tool for proving our main results. Sections 4 and 5 are the heart of the paper, in which we prove the change of variable and pointwise multiplication results already discussed in the introduction section. For the convenience of the reader, we also discuss the onedimensional versions of the results in Sects. 4 and 5 in detail.

\subsection{Notation}

As usual, $\mathbb{N}$ denotes the natural numbers, $\mathbb{N}_{0}=\mathbb{N} \cup\{0\}, \mathbb{Z}$ denotes the integers, $\mathbb{R}$ the real numbers, and $\mathbb{C}$ the complex numbers. By $\mathbb{T}:=\mathbb{R} / \mathbb{Z}$ we denote the torus represented with the interval $[0,1]$ in $\mathbb{R}$. The letter $d$ is always reserved for the underlying dimension in $\mathbb{R}^{d}, \mathbb{Z}^{d}$, etc., and by $[d]$ we mean $[d]=\{1, \ldots, d\}$. We denote by $\langle x, y\rangle$ or $x \cdot y$ the usual Euclidean inner product in $\mathbb{R}^{d}$ or $\mathbb{C}^{d}$. For $0<p \leq \infty$ and $x \in \mathbb{R}^{d}$, we write $|x|_{p}=\left(\sum_{i=1}^{d}\left|x_{i}\right|^{p}\right)^{1 / p}$ with the usual modification in the case $p=\infty$. By $\left(x_{1}, \ldots, x_{d}\right)>0$, we mean that each coordinate is positive. As usual, we decompose $x \in \mathbb{R}$ in $x=\lfloor x\rfloor+\{x\}$, where $0 \leq\{x\}<1$ (resp. $\{x\}$ for $x \in \mathbb{R}^{d}$ is meant component-wise).

If $X$ and $Y$ are two (quasi-)normed spaces, the (quasi-)norm of an element $x$ in $X$ will be denoted by $\|x\|_{X}$. The symbol $X \hookrightarrow Y$ indicates that the identity operator is continuous. For two sequences $a_{n}$ and $b_{n}$, we will write $a_{n} \lesssim b_{n}$ if there exists a 
constant $c>0$ such that $a_{n} \leq c b_{n}$ for all $n$. We will write $a_{n} \asymp b_{n}$ if $a_{n} \lesssim b_{n}$ and $b_{n} \lesssim a_{n}$.

\section{Tools from Fourier Analysis}

In this section, we will collect the required tools from Fourier analysis.

\subsection{Preliminaries}

Let $L_{p}=L_{p}\left(\mathbb{R}^{d}\right), 0<p \leq \infty$, be the space of all functions $f: \mathbb{R}^{d} \rightarrow \mathbb{C}$ such that

$$
\|f\|_{p}:=\left(\int_{\mathbb{R}^{d}}|f(x)|^{p} \mathrm{~d} x\right)^{1 / p}<\infty
$$

with the usual modification if $p=\infty$. We will also need $L_{p}$-spaces on compact domains $\Omega \subset \mathbb{R}^{d}$ instead of $\mathbb{R}^{d}$. We write $\|f\|_{L_{p}(\Omega)}$ for the corresponding (restricted) $L_{p}$-norm.

For $k \in \mathbb{N}_{0}$, we denote by $C_{0}^{k}\left(\mathbb{R}^{d}\right)$ the collection of all compactly supported functions $\varphi$ on $\mathbb{R}^{d}$ that have $D^{\alpha} \varphi$ uniformly continuous on $\mathbb{R}^{d}$ for $|\alpha|_{\infty} \leq k$.

Additionally, we define the spaces of infinitely differentiable functions $C^{\infty}\left(\mathbb{R}^{d}\right)$ and infinitely differentiable functions with compact support $C_{0}^{\infty}\left(\mathbb{R}^{d}\right)$ as well as the Schwartz space $\mathcal{S}=\mathcal{S}\left(\mathbb{R}^{d}\right)$ of all rapidly decaying infinitely differentiable functions on $\mathbb{R}^{d}$; i.e.,

$$
\mathcal{S}:=\left\{\varphi \in C^{\infty}\left(\mathbb{R}^{d}\right):\|\varphi\|_{k, \ell}<\infty \quad \text { for all } k, \ell \in \mathbb{N}\right\}
$$

and

$$
\|\varphi\|_{k, \ell}:=\left\|(1+|\cdot|)^{k} \sum_{|\alpha|_{1} \leq \ell}\left|D^{\alpha} \varphi(\cdot)\right|\right\|_{\infty}, \quad \alpha \in \mathbb{N}_{0}^{d} .
$$

The space $\mathcal{S}^{\prime}\left(\mathbb{R}^{d}\right)$, the topological dual of $\mathcal{S}\left(\mathbb{R}^{d}\right)$, is also referred to as the set of tempered distributions on $\mathbb{R}^{d}$. Indeed, a linear mapping $f: \mathcal{S}\left(\mathbb{R}^{d}\right) \rightarrow \mathbb{C}$ belongs to $\mathcal{S}^{\prime}\left(\mathbb{R}^{d}\right)$ if and only if there exist numbers $k, \ell \in \mathbb{N}$ and a constant $c=c_{f}$ such that

$$
|f(\varphi)| \leq c_{f}\|\varphi\|_{k, \ell}
$$

for all $\varphi \in \mathcal{S}\left(\mathbb{R}^{d}\right)$. The space $\mathcal{S}^{\prime}\left(\mathbb{R}^{d}\right)$ is equipped with the weak-star topology.

For $f \in L_{1}\left(\mathbb{R}^{d}\right)$, we define the Fourier transform

$$
\mathcal{F} f(\xi)=\int_{\mathbb{R}^{d}} f(y) e^{-2 \pi i\langle\xi, y\rangle} \mathrm{d} y, \quad \xi \in \mathbb{R}^{d},
$$


and the corresponding inverse Fourier transform $\mathcal{F}^{-1} f(\xi)=\mathcal{F} f(-\xi)$. As usual, the Fourier transform can be extended to $\mathcal{S}^{\prime}\left(\mathbb{R}^{d}\right)$ by $(\mathcal{F} f)(\varphi):=f(\mathcal{F} \varphi)$, where $f \in \mathcal{S}^{\prime}\left(\mathbb{R}^{d}\right)$ and $\varphi \in \mathcal{S}\left(\mathbb{R}^{d}\right)$. The mapping $\mathcal{F}: \mathcal{S}^{\prime}\left(\mathbb{R}^{d}\right) \rightarrow \mathcal{S}^{\prime}\left(\mathbb{R}^{d}\right)$ is a bijection.

The convolution $\varphi * \psi$ of two square-integrable functions $\varphi, \psi$ is defined via the integral

$$
(\varphi * \psi)(x)=\int_{\mathbb{R}^{d}} \varphi(x-y) \psi(y) \mathrm{d} y
$$

If $\varphi, \psi \in \mathcal{S}\left(\mathbb{R}^{d}\right)$, then $\varphi * \psi$ still belongs to $\mathcal{S}\left(\mathbb{R}^{d}\right)$. In fact, we have $\varphi * f \in \mathcal{S}\left(\mathbb{R}^{d}\right)$ even if $\varphi \in \mathcal{S}\left(\mathbb{R}^{d}\right)$ and $f \in L_{1}\left(\mathbb{R}^{d}\right)$. The convolution can be extended to $\mathcal{S}\left(\mathbb{R}^{d}\right) \times \mathcal{S}^{\prime}\left(\mathbb{R}^{d}\right)$ via $(\varphi * f)(x)=f(\varphi(x-\cdot))$. It makes sense pointwise and is a $C^{\infty}$-function in $\mathbb{R}^{d}$.

\subsection{Maximal Inequalities}

Let us provide here the maximal inequalities for the Hardy-Littlewood and Peetre maximal functions, respectively, which are essential for the proofs of Theorems 4.1 and 4.2. For further details, we refer to [50, 1.2, 1.3] or [29, Chapt. 2] .

For a locally integrable function $f: \mathbb{R}^{d} \rightarrow \mathbb{C}$, we denote by $M f(x)$ the HardyLittlewood maximal function defined by

$$
(M f)(x)=\sup _{x \in Q} \frac{1}{|Q|} \int_{Q}|f(y)| \mathrm{d} y, \quad x \in \mathbb{R}^{d},
$$

where the supremum is taken over all cubes with sides parallel to the coordinate axes containing $x$. A vector-valued generalization of the classical Hardy-Littlewood maximal inequality is due to Fefferman and Stein [11].

Theorem 2.1 For $1<p<\infty$ and $1<\theta \leq \infty$, there exists a constant $c>0$, such that

$$
\left\|\left(\sum_{\ell \in I}\left|M f_{\ell}\right|^{\theta}\right)^{1 / \theta}\right\|_{p} \leq c\left\|\left(\sum_{\ell \in I}\left|f_{\ell}\right|^{\theta}\right)^{1 / \theta}\right\|_{p}
$$

holds for all sequences $\left\{f_{\ell}\right\}_{\ell \in I}$ of locally Lebesgue-integrable functions on $\mathbb{R}^{d}$ :

We require a direction-wise version of (2.1):

$$
\left(M_{i} f\right)(x)=\sup _{s>0} \frac{1}{2 s} \int_{x_{i}-s}^{x_{i}+s}\left|f\left(x_{1}, \ldots, x_{i-1}, t, x_{i+1}, \ldots, x_{d}\right)\right| \mathrm{d} t, \quad x \in \mathbb{R}^{d}
$$

We denote the composition of these operators by $M_{e}=\prod_{i \in e} M_{i}$, where $e$ is a subset of $[d]$ and $M_{\ell} M_{k}$ has to be interpreted as $M_{\ell} \circ M_{k}$. The following version of the Fefferman-Stein maximal inequality is due Stöckert [32]. 
Theorem 2.2 For $1<p<\infty$ and $1<\theta \leq \infty$, there exists a constant $c>0$, such that for any $i \in[d]$,

$$
\left\|\left(\sum_{\ell \in I}\left|M_{i} f_{\ell}\right|^{\theta}\right)^{1 / \theta}\right\|_{p} \leq c\left\|\left(\sum_{\ell \in I}\left|f_{\ell}\right|^{\theta}\right)^{1 / \theta}\right\|_{p}
$$

holds for all sequences $\left\{f_{\ell}\right\}_{\ell \in I}$ of locally Lebesgue-integrable functions on $\mathbb{R}^{d}$.

Iteration of this theorem yields a similar boundedness property for the operator $M_{[d]}$. The following construction of a maximal function is due to Peetre, Fefferman, and Stein. Let $b=\left(b_{1}, \ldots, b_{d}\right)>0, a>0$, and $f \in L_{1}\left(\mathbb{R}^{d}\right)$ with $\mathcal{F} f$ compactly supported. We define the Peetre maximal function $P_{b, a} f$ by

$$
P_{b, a} f(x)=\sup _{z \in \mathbb{R}^{d}} \frac{|f(x-z)|}{\left(1+\left|b_{1} z_{1}\right|\right)^{a} \cdot \ldots \cdot\left(1+\left|b_{d} z_{d}\right|\right)^{a}}
$$

Lemma 2.3 Let $\Omega \subset \mathbb{R}^{d}$ be a compact set. Further, let $a>0$ and $\alpha=\left(\alpha_{1}, \ldots, \alpha_{d}\right) \in$ $\mathbb{N}_{0}^{d}$. Then there exist two constants $c_{1}, c_{2}>0$ (independently of $f$ ) such that

$$
\begin{aligned}
P_{(1, \ldots, 1), a}\left(D^{\alpha} f\right)(x) & \leq c_{1} P_{(1, \ldots, 1), a} f(x) \\
& \leq c_{2}\left(M_{d}\left(M_{d-1}\left(\ldots\left(M_{1}|f|^{1 / a}\right) \ldots\right)\right)\right)^{a}(x)
\end{aligned}
$$

holds for all $f \in L_{1}\left(\mathbb{R}^{d}\right)$ with $\operatorname{supp}(\mathcal{F} f) \subset \Omega$ and all $x \in \mathbb{R}^{d}$. The constants $c_{1}, c_{2}$ depend on $\Omega$.

We will need the vector-valued Peetre maximal inequality, which is a direct consequence of Lemma 2.3 together with Theorem 2.2.

Theorem 2.4 Let $0<p<\infty, 0<\theta \leq \infty$ and $a>\max \{1 / p, 1 / \theta\}$. Further, let $b^{\ell}=\left(b_{1}^{\ell}, \ldots, b_{d}^{\ell}\right)>0$ for $\ell \in I$ and $\Omega=\left\{\Omega_{\ell}\right\}_{\ell \in I}$, such that

$$
\Omega_{\ell} \subset\left[-b_{1}^{\ell}, b_{1}^{\ell}\right] \times \ldots \times\left[-b_{d}^{\ell}, b_{d}^{\ell}\right]
$$

is compact for $\ell \in I$. Then there is a constant $C>0$ (independently of $f$ and $\Omega$ ) such that

$$
\left\|\left(\sum_{\ell \in I}\left|P_{b^{\ell}, a} f_{\ell}\right|^{\theta}\right)^{1 / \theta}\right\|_{p} \leq C\left\|\left(\sum_{\ell \in I}\left|f_{\ell}\right|^{\theta}\right)^{1 / \theta}\right\|_{p}
$$

holds for all systems $f=\left\{f_{\ell}\right\}_{\ell \in I}$ with $\operatorname{supp}\left(\mathcal{F} f_{\ell}\right) \subset \Omega_{\ell}, \ell \in I$.

\section{Function Spaces of Dominating Mixed Smoothness}

In this section, we introduce the function spaces under consideration, namely, the Besov and Triebel-Lizorkin spaces of dominating mixed smoothness. Note that the 
Sobolev spaces $\mathbf{W}_{p}^{s}$ of mixed smoothness appear as a special case of the TriebelLizorkin spaces; namely, $\mathbf{W}_{p}^{s}=\mathbf{F}_{p, 2}^{s}$ if $1<p<\infty$ and $s>0$. There are several equivalent characterizations of these spaces, see, e.g., [50]. We begin with the usual definition of the spaces using a dyadic decomposition of unity on the Fourier side, following Triebel [43]. Afterwards, we present an equivalent characterization by mixed iterated differences. To begin with, we introduce the concept of smooth dyadic decomposition of unity. Let $\left\{\eta_{j}\right\}_{j \in \mathbb{N}_{0}} \subset C_{0}^{\infty}(\mathbb{R})$ such that:

(i) $\operatorname{supp}\left(\eta_{0}\right) \subset\{t:|t| \leq 2\}$,

(ii) $\operatorname{supp}\left(\eta_{j}\right) \subset\left\{t: 2^{j-1} \leq|t| \leq 2^{j+1}\right\}, j \in \mathbb{N}$,

(iii) for all $\ell \in \mathbb{N}_{0}$, it holds that $\sup _{t, j} 2^{j \ell}\left|D^{\ell} \eta_{j}(t)\right| \leq c_{\ell}<\infty$, and

(iv) $\sum_{j \in \mathbb{N}_{0}} \eta_{j}(t)=1$ for all $t \in \mathbb{R}$.

Such a family of functions obviously exists. To approach function spaces with dominating mixed smoothness, we define the family $\left\{\eta_{j}\right\}_{j \in \mathbb{N}_{0}^{d}}$ of $d$-variate functions as the tensor products

$$
\eta_{j}(x)=\prod_{i=1}^{d} \eta_{j_{i}}\left(x_{i}\right)
$$

where $j=\left(j_{1}, \ldots, j_{d}\right) \in \mathbb{N}_{0}^{d}$ and $x=\left(x_{1}, \ldots, x_{d}\right) \in \mathbb{R}^{d}$.

\subsection{Spaces on $\mathbb{R}^{d}$}

Let us start with the definition of the function spaces $\mathbf{A}_{p, \theta}^{s}=\mathbf{A}_{p, \theta}^{s}\left(\mathbb{R}^{d}\right)$ with $\mathbf{A} \in\{\mathbf{B}, \mathbf{F}\}$ defined on the entire $\mathbb{R}^{d}$.

Definition 3.1 (Besov space) Let $0<p, \theta \leq \infty, s \in \mathbb{R}$, and $\left\{\eta_{j}\right\}_{j \in \mathbb{N}_{0}^{d}}$ be as above. The Besov space of dominating mixed smoothness $\mathbf{B}_{p, \theta}^{s}=\mathbf{B}_{p, \theta}^{s}\left(\mathbb{R}^{d}\right)$ is the set of all $f \in \mathcal{S}^{\prime}\left(\mathbb{R}^{d}\right)$ such that

$$
\|f\|_{\mathbf{B}_{p, \theta}^{s}}:=\left(\sum_{j \in \mathbb{N}_{0}^{d}} 2^{s|j|_{1} \theta}\left\|\mathcal{F}^{-1}\left[\eta_{j} \mathcal{F} f\right]\right\|_{p}^{\theta}\right)^{1 / \theta}<\infty
$$

with the usual modification for $\theta=\infty$.

Definition 3.2 (Triebel-Lizorkin space) Let $0<p<\infty, 0<\theta \leq \infty, s \in \mathbb{R}$, and $\left\{\eta_{j}\right\}_{j \in \mathbb{N}_{0}^{d}}$ be as above. The Triebel-Lizorkin space of dominating mixed smoothness $\mathbf{F}_{p, \theta}^{s}=\mathbf{F}_{p, \theta}^{s}\left(\mathbb{R}^{d}\right)$ is the set of all $f \in \mathcal{S}^{\prime}\left(\mathbb{R}^{d}\right)$ such that

$$
\|f\|_{\mathbf{F}_{p, \theta}^{s}}:=\left\|\left(\sum_{j \in \mathbb{N}_{0}^{d}} 2^{s|j|_{1} \theta}\left|\mathcal{F}^{-1}\left[\eta_{j} \mathcal{F} f\right]\right|^{\theta}\right)^{1 / \theta}\right\|_{p}<\infty
$$

with the usual modification for $\theta=\infty$. 
Remark 3.3 In the special case $\theta=2$ and $1<p<\infty$, we put $\mathbf{W}_{p}^{s}:=\mathbf{F}_{p, 2}^{s}$, which denotes the Sobolev spaces of dominating mixed smoothness. It is well known (cf. [29, Chapt. 2] for $d=2$ ), that in case $s \in \mathbb{N}_{0}$, the spaces $\mathbf{W}_{p}^{s}$ can be equivalently normed by

$$
\|f\|_{\mathbf{W}_{p}^{s}} \asymp\left(\sum_{\substack{\alpha \in \mathbb{N}_{0}^{d} \\|\alpha|_{\infty} \leq s}}\left\|D^{\alpha} f\right\|_{p}^{p}\right)^{1 / p} .
$$

Remark 3.4 Different choices of smooth dyadic decompositions of unity $\left\{\eta_{j}\right\}_{j \in \mathbb{N}_{0}}$ lead to equivalent (quasi-)norms.

The next lemma collects some frequently used embedding properties of the spaces.

Lemma 3.5 Let $0<p<\infty, 0<\theta \leq \infty, s \in \mathbb{R}$, and $\mathbf{A} \in\{\mathbf{B}, \mathbf{F}\}$.

(i) If $s>\max \{1 / p-1,0\}$, then

$$
\mathbf{A}_{p, \theta}^{s} \hookrightarrow L_{\max \{p, 1\}}\left(\mathbb{R}^{d}\right) .
$$

(ii) If $s>1 / p$, then

$$
\mathbf{A}_{p, \theta}^{s} \hookrightarrow C\left(\mathbb{R}^{d}\right)
$$

Proof For a proof, we refer to [29, Chapt. 2].

Lemma 3.5(ii) gives a sufficient condition for $\mathbf{A}_{p, \theta}^{s}$ to consist only of continuous functions. This is of particular importance for the analysis of cubature rules, since function evaluations have to be defined reasonably.

\subsection{Spaces on the $d$-torus and Nonperiodic Functions on the Cube $[0,1]^{d}$}

Let $\mathbb{T}^{d}=[0,1]^{d}$ and $f: \mathbb{T}^{d} \rightarrow \mathbb{C}$ be a function defined on $\mathbb{R}^{d}$ and supposed to be 1-periodic in every component. We will define periodicity in a wider sense. A distribution $f \in \mathcal{S}^{\prime}\left(\mathbb{R}^{d}\right)$ is a periodic distribution from $\mathcal{S}_{\pi}^{\prime}\left(\mathbb{R}^{d}\right)$ if and only if

$$
f(\varphi)=f(\varphi(\cdot+k)), \quad \varphi \in \mathcal{S}\left(\mathbb{R}^{d}\right), k \in \mathbb{Z}^{d} .
$$

Now we define

$$
\mathbf{A}_{p, \theta}^{s}\left(\mathbb{T}^{d}\right)=\left\{f \in \mathcal{S}_{\pi}^{\prime}\left(\mathbb{R}^{d}\right):\|f\|_{\mathbf{A}_{p, \theta}^{s}\left(\mathbb{T}^{d}\right)}<\infty\right\},
$$

with

$$
\|f\|_{\mathbf{B}_{p, \theta}^{s}\left(\mathbb{T}^{d}\right)}:=\left(\sum_{j \in \mathbb{N}_{0}^{d}} 2^{s|j|_{1} \theta}\left\|\mathcal{F}^{-1}\left[\eta_{j} \mathcal{F} f\right]\right\|_{L_{p}\left(\mathbb{T}^{d}\right)}^{\theta}\right)^{1 / \theta}
$$


and

$$
\|f\|_{\mathbf{F}_{p, \theta}^{s}\left(\mathbb{T}^{d}\right)}:=\left\|\left(\sum_{j \in \mathbb{N}_{0}^{d}} 2^{s|j|_{1} \theta}\left|\mathcal{F}^{-1}\left[\eta_{j} \mathcal{F} f\right]\right|^{\theta}\right)^{1 / \theta}\right\|_{L_{p}\left(\mathbb{T}^{d}\right)},
$$

respectively. Note that we replace the $L_{p}\left(\mathbb{R}^{d}\right)$-norms by $L_{p}\left(\mathbb{T}^{d}\right)$-norms. If we denote by $\left\{c_{k}(f)\right\}_{k \in \mathbb{Z}^{d}}$ the Fourier coefficients of $f$, then we could write

$$
\mathcal{F}^{-1}\left[\eta_{j} \mathcal{F} f\right](x)=\sum_{k \in \mathbb{Z}^{d}} c_{k}(f) \eta_{j}(k) e^{2 \pi i k x},
$$

see [29, p. 150].

Next we turn to the subspace of functions that are supported in a compact set $\Omega$. That is, we define for $s>\sigma_{p}=\max \{1 / p-1,0\}$ the spaces

$$
\stackrel{\circ}{\text { A }}_{p, \theta}(\Omega):=\left\{f \in \mathbf{A}_{p, \theta}^{s}\left(\mathbb{R}^{d}\right): \operatorname{supp}(f) \subset \Omega\right\} .
$$

Due to Lemma 3.5(i), this definition is reasonable. If $\Omega=[0,1]^{d}$, we write $\AA_{p, \theta}^{s}$ instead of $\AA_{p, \theta}^{s}\left([0,1]^{d}\right)$. The space $\AA_{p, \theta}^{s}$ can be interpreted as subspaces of $\mathbf{A}_{p, \theta}^{s}\left(\mathbb{T}^{d}\right)$. Finally, we define the spaces $\mathbf{A}_{p, \theta}^{s}\left([0,1]^{d}\right)$. For large enough $s$, i.e., $s>\sigma_{p}$, we define $\mathbf{A}_{p, \theta}^{s}\left([0,1]^{d}\right)$ as the collection of all functions $f \in \mathbf{A}_{p, \theta}^{s}$ restricted to the unit cube $[0,1]^{d}$; i.e.,

$$
\mathbf{A}_{p, \theta}^{s}\left([0,1]^{d}\right):=\left\{f=\left.g\right|_{[0,1]^{d}}: g \in \mathbf{A}_{p, \theta}^{s}\left(\mathbb{R}^{d}\right)\right\}
$$

The (quasi-)norm on these spaces is usually given by

$$
\|f\|_{\mathbf{A}_{p, \theta}^{s}\left([0,1]^{d}\right)}:=\inf \left\{\|g\|_{\mathbf{A}_{p, \theta}^{s}}: g \in \mathbf{A}_{p, \theta}^{s} \text { and }\left.g\right|_{[0,1]^{d}}=f\right\} .
$$

\subsection{Characterization by Mixed Iterated Differences}

In this section, we will provide a characterization of the above defined spaces that is actually the classical way of defining them, see for instance Nikol'skij [25], Amanov [1], Schmeißer, Triebel [29], Temlyakov [36] and the references therein. For us it will be convenient to replace the classical moduli of continuity by so-called rectangular means of differences, see (3.3) below. This is is the counterpart of the ball means of differences for isotropic spaces, see [40, Thm. 2.5.11].

Let us first recall the basic concepts. For univariate functions $f: \mathbb{R} \rightarrow \mathbb{C}$, the $m$ th difference operator $\Delta_{h}^{m}$ is defined as

$$
\Delta_{h}^{m}(f, t):=\sum_{j=0}^{m}(-1)^{m-j}\left(\begin{array}{c}
m \\
j
\end{array}\right) f(t+j h), \quad t \in \mathbb{R}, h \in \mathbb{R}
$$


Let $e$ be any subset of $[d]=\{1, \ldots, d\}$. For multivariate functions $f: \mathbb{R}^{d} \rightarrow \mathbb{C}$ and $h \in \mathbb{R}^{d}$, the mixed $(m, e)$ th difference operator $\Delta_{h}^{m, e}$ is defined as

$$
\Delta_{h}^{m, e}:=\prod_{i \in e} \Delta_{h_{i}, i}^{m} \text { and } \Delta_{h}^{m, \emptyset}=\mathrm{Id}
$$

where Id $f=f$ and $\Delta_{h_{i}, i}^{m}$ is the univariate operator applied to the $i$ th coordinate of $f$ with the other variables kept fixed. Whenever we write the "product" $\prod_{i \in e} A_{i}$ of operators $A_{i}$ we mean the concatenation of the $A_{i}$, see also the small paragraph before Theorem 2.2 above.

Now we define the so-called rectangular means of differences by

$$
\mathcal{R}_{m}^{e}(f, t, x):=\int_{[-1,1]^{d}}\left|\Delta_{\left(h_{1} t_{1}, \ldots, h_{d} t_{d}\right)}^{m, e}(f, x)\right| \mathrm{d} h, \quad x \in \mathbb{R}^{d}, t \in(0,1]^{d} .
$$

For $j \in \mathbb{N}_{0}^{d}$, we put $e(j)=\left\{i: j_{i} \neq 0\right\}$ and $e_{0}(j)=[d] \backslash e(j)$. We have the following theorems.

Theorem 3.6 Let $0<p, \theta \leq \infty$ and $s>\sigma_{p}$. Further, let $m \in \mathbb{N}$ be a natural number with $m>s$. Then

$$
\|f\|_{\mathbf{B}_{p, \theta}^{s}} \asymp\|f\|_{\mathbf{B}_{p, \theta}^{s}}^{(m)}, \quad f \in L_{1}\left(\mathbb{R}^{d}\right)
$$

where

$$
\|f\|_{\mathbf{B}_{p, \theta}^{s}}^{(m)}:=\left(\sum_{j \in \mathbb{N}_{0}^{d}} 2^{s|j|_{1} \theta}\left\|\mathcal{R}_{m}^{e(j)}\left(f, 2^{-j}, \cdot\right)\right\|_{p}^{\theta}\right)^{1 / \theta}
$$

In case $\theta=\infty$, the sum above is replaced by the supremum over $j$. Here $2^{-j}:=$ $\left(2^{-j_{1}}, \ldots, 2^{-j_{d}}\right)$.

Theorem 3.7 Let $0<p<\infty, 0<\theta \leq \infty$, and $s>\sigma_{p, \theta}$. Further, let $m \in \mathbb{N}$ be a natural number with $m>s$. Then

$$
\|f\|_{\mathbf{F}_{p, \theta}^{s}} \asymp\|f\|_{\mathbf{F}_{p, \theta}^{s}}^{(m)}, \quad f \in L_{1}\left(\mathbb{R}^{d}\right),
$$

where

$$
\|f\|_{\mathbf{F}_{p, \theta}^{s}}^{(m)}:=\left\|\left(\sum_{j \in \mathbb{N}_{0}^{d}} 2^{s|j|_{1} \theta} \mathcal{R}_{m}^{e(j)}\left(f, 2^{-j}, \cdot\right)^{\theta}\right)^{1 / \theta}\right\|_{p} .
$$

Since the characterizations of the spaces $\mathbf{B}_{p, \theta}^{s}$ and $\mathbf{F}_{p, \theta}^{s}$ that are given in Theorems 3.6 and 3.7 are, as far as we know, not proved in the literature, we will give a proof here for the convenience of the reader. 
The statements in Theorems 3.6 and 3.7 are "discretized versions" of the characterizations given in [47]. In addition, the range of the sum $\sum_{j \in \mathbb{N}_{0}^{d}}$ is crucial. Here, we mainly have to establish the relation $\|f\|_{\mathbf{F}_{p, \theta}^{s}} \asymp\|f\|_{\mathbf{F}_{p, \theta}^{s}}^{(m)}$, which is done via the characterization for the spaces $\mathbf{B}_{p, \theta}^{s}$ and $\mathbf{F}_{p, \theta}^{s}$ via local means, see [49,50]. Given a function $\Psi: \mathbb{R} \rightarrow \mathbb{C}$, we denote by $L_{\Psi} \in \mathbb{N}$ the maximal number such that $\Psi$ has vanishing moments; i.e.,

$$
\int_{\mathbb{R}} t^{\alpha} \Psi(t) \mathrm{d} t=0, \quad \alpha=0, \ldots, L_{\Psi}
$$

Let $\Psi_{0} \in \mathcal{S}(\mathbb{R})$ be a function satisfying the following conditions:

$$
\int_{\mathbb{R}} \Psi_{0}(t) \mathrm{d} t \neq 0 \text { and } L_{\Psi} \geq R \text { for } \Psi(t)=\Psi_{0}(t)-\frac{1}{2} \Psi_{0}(t / 2),
$$

for some $R \in \mathbb{N}$. For $k \in \mathbb{N}$, we put $\Psi_{k}(t)=2^{k} \Psi\left(2^{k} t\right), t \in \mathbb{R}$. If $j \in \mathbb{N}_{0}^{d}$, we write

$$
\Psi_{j}\left(x_{1}, \ldots, x_{d}\right)=\Psi_{j_{1}}\left(x_{1}\right) \cdot \ldots \cdot \Psi_{j_{d}}\left(x_{d}\right), \quad x \in \mathbb{R}^{d}
$$

We have the following proposition, see [50, Thm. 1.23] and [49].

Proposition 3.8 Let $s \in \mathbb{R}$ and $\Psi_{0}$ be given by (3.4) with $R+1>s$.

(i) Let $0<p, \theta \leq \infty$. Then $\mathbf{B}_{p, \theta}^{s}$ is the collection of all $f \in \mathcal{S}^{\prime}\left(\mathbb{R}^{d}\right)$ such that

$$
\|f\|_{\mathbf{B}_{p, \theta}^{s}}=\left(\sum_{j \in \mathbb{N}_{0}^{d}} 2^{|j|_{1} s \theta}\left\|\Psi_{j} * f\right\|_{p}^{\theta}\right)^{1 / \theta}<\infty .
$$

(ii) Let $0<p<\infty$ and $0<\theta \leq \infty$. Then $\mathbf{F}_{p, \theta}^{s}$ is the collection of all $f \in \mathcal{S}^{\prime}\left(\mathbb{R}^{d}\right)$ such that

$$
\|f\|_{\mathbf{F}_{p, \theta}^{s}}=\left\|\left(\sum_{j \in \mathbb{N}_{0}^{d}} 2^{|j|_{1} s \theta}\left|\Psi_{j} * f\right|^{\theta}\right)^{1 / \theta}\right\|_{p}<\infty
$$

Now we are in a position to prove Theorem 3.7. The proof of Theorem 3.6 is similar but technically less involved.

Proof (Proof of Theorem 3.7) Step 1 We prove the inequality

$$
\|f\|_{\mathbf{F}_{p, \theta}^{s}}^{(m)} \lesssim\|f\|_{\mathbf{F}_{p, \theta}^{s}} .
$$

This inequality is a consequence of [47, Thm. 3.4.1]. Indeed, from the equivalent norm

$$
\|f\|_{\mathbf{F}_{p, \theta}^{s}} \asymp\|f\|_{p}+\sum_{e \subset[d], e \neq \emptyset}\left\|\left(\int_{(0, \infty)|e|}\left(\prod_{i \in e} t_{i}^{-s \theta}\right) \mathcal{R}_{m}^{e}(f, t, \cdot)^{\theta} \prod_{i \in e} \frac{\mathrm{d} t_{i}}{t_{i}}\right)^{1 / \theta}\right\|_{p}
$$


see [47, Thm. 3.4.1], we have

$$
\|f\|_{\mathbf{F}_{p, \theta}^{s}} \gtrsim\|f\|_{p}+\sum_{e \subset[d], e \neq \emptyset}\left\|\left(\int_{(0,1)^{|e|}}\left(\prod_{i \in e} t_{i}^{-s \theta}\right) \mathcal{R}_{m}^{e}(f, t, \cdot)^{\theta} \prod_{i \in e} \frac{\mathrm{d} t_{i}}{t_{i}}\right)^{1 / \theta}\right\|_{p} .
$$

Discretizing the right-hand side of the above inequality, we obtain

$$
\begin{aligned}
& \left\|\left(\sum_{j \in \mathbb{N}_{0}^{d}} 2^{|j|_{1} s \theta} \mathcal{R}_{m}^{e(j)}\left(f, 2^{-j}, \cdot\right)^{\theta}\right)^{1 / \theta}\right\|_{p} \\
& \lesssim\|f\|_{p}+\sum_{e \subset[d], e \neq \emptyset}\left\|\left(\int_{(0,1)^{|e|}}\left(\prod_{i \in e} t_{i}^{-s \theta}\right) \mathcal{R}_{m}^{e}(f, t, \cdot)^{\theta} \prod_{i \in e} \frac{\mathrm{d} t_{i}}{t_{i}}\right)^{1 / \theta}\right\|_{p},
\end{aligned}
$$

which implies (3.5).

Step 2 We prove the reverse inequality in (3.5). This time we rely on the intrinsic characterization of $\mathbf{F}_{p, \theta}^{s}$ via local means, see Proposition 3.8.

Substep 2.1 Preparation. According to [41,3.3.1, 3.3.2], for $m \in \mathbb{N}$, there exist function $\psi(t)$ with

$$
\operatorname{supp}(\psi) \subset\left(-\frac{1}{m}, \frac{1}{m}\right) \quad \text { and } \quad \int_{\mathbb{R}} \psi(t) \mathrm{d} t=1
$$

such that

$$
\Psi_{0}(t)=\frac{(-1)^{m+1}}{m !} \sum_{u=1}^{m} \sum_{v=1}^{m}(-1)^{u+v}\left(\begin{array}{c}
m \\
u
\end{array}\right)\left(\begin{array}{c}
m \\
v
\end{array}\right) v^{m}(u v)^{-1} \psi\left(\frac{t}{u v}\right), t \in \mathbb{R},
$$

satisfies (3.4) with $R=m$. Putting

$$
\eta(t)=\frac{(-1)^{m+1}}{m !} \sum_{v=1}^{m}(-1)^{m-v}\left(\begin{array}{c}
m \\
v
\end{array}\right) v^{m} v^{-1} \psi\left(\frac{t}{v}\right), t \in \mathbb{R},
$$

then we have $\operatorname{supp}(\eta) \in(-1,1)$, and moreover,

$$
\Psi_{0}(t)=\sum_{u=1}^{m}(-1)^{m-u}\left(\begin{array}{l}
m \\
u
\end{array}\right) u^{-1} \eta\left(\frac{t}{u}\right)
$$


A simple computation gives for a univariate function $g$,

$$
\begin{aligned}
\Psi_{0} * g(t) & =\int_{\mathbb{R}} \Psi_{0}(h) g(t-h) \mathrm{d} h \\
& =\int_{\mathbb{R}} \eta(-h) \sum_{u=1}^{m}(-1)^{m-u}\left(\begin{array}{l}
m \\
u
\end{array}\right) g(t+u h) \mathrm{d} h \\
& =\int_{-1}^{1} \eta(-h)\left[\Delta_{h}^{m} g(t)-(-1)^{m} g(t)\right] \mathrm{d} h
\end{aligned}
$$

and

$$
\begin{aligned}
\Psi_{k} * g(t) & =\int_{\mathbb{R}} \Psi(h) g\left(t-2^{-k} h\right) \mathrm{d} h \\
& =\int_{\mathbb{R}}\left[\eta(-h)-\frac{1}{2} \eta\left(-\frac{h}{2}\right)\right] \Delta_{2^{-k} h}^{m} g(t) \mathrm{d} h \\
& =\int_{-1}^{1} \eta(-h)\left[\Delta_{2^{-k} h}^{m}-\Delta_{2^{-k+1} h}^{m}\right] g(t) \mathrm{d} h .
\end{aligned}
$$

Substep 2.2 We define the function $\eta(\cdot)$ on $\mathbb{R}^{d}$ as $d$-fold tensor product; i.e.,

$$
\eta(x)=\eta\left(x_{1}\right) \cdot \ldots \cdot \eta\left(x_{d}\right), \quad x=\left(x_{1}, \ldots, x_{d}\right) \in \mathbb{R}^{d} .
$$

From (3.6) and (3.7), we have for $j \in \mathbb{N}_{0}^{d}$,

$$
\begin{aligned}
\Psi_{j} * f(x)= & \int_{[-1,1]^{d}} \eta(-h)\left[\prod_{i \in e(j)}\left(\Delta_{2^{-j_{i} h_{i}, i}}^{m}-\Delta_{2^{-j_{i}+1} h_{i}, i}^{m}\right)\right. \\
& \left.\times \prod_{i \in e_{0}(j)}\left(\Delta_{h_{i}, i}^{m}-(-1)^{m} \mathrm{Id}\right)\right] f(x) \mathrm{d} h .
\end{aligned}
$$

Then we obtain

$$
\left|\Psi_{j} * f(x)\right| \leq \sum_{u \in\{0,1\}^{d}} \mathcal{R}_{m}^{e(j+u)}\left(f, 2^{-j+u}, x\right)
$$

which leads to

$$
\begin{aligned}
\left\|\left(\sum_{j \in \mathbb{N}_{0}^{d}} 2^{s|j|{ }_{1} \theta}\left|\Psi_{j} * f\right|^{\theta}\right)^{1 / \theta}\right\|_{p} & \lesssim\left\|\left(\sum_{j \in \mathbb{N}_{0}^{d}} 2^{s|j|_{1} \theta} \sum_{u \in\{0,1\}^{d}} \mathcal{R}_{m}^{e(j+u)}\left(f, 2^{-j+u}, \cdot\right)^{\theta}\right)^{1 / \theta}\right\|_{p} \\
& \lesssim\left\|\left(\sum_{j \in \mathbb{N}_{0}^{d}} 2^{\left.s|j|\right|_{1} \theta} \mathcal{R}_{m}^{e(j)}\left(f, 2^{-j}, \cdot\right)^{\theta}\right)^{1 / \theta}\right\|_{p} .
\end{aligned}
$$

In view of Proposition 3.8 we finish the proof. 


\section{Change of Variables}

Let $\varphi \in C_{0}^{k}(\mathbb{R}) \operatorname{such}$ that $\operatorname{supp}(\varphi) \subset[0,1], \int_{0}^{1} \varphi(t) \mathrm{d} t=1, \varphi(t)>0$ on $(0,1)$, and $\varphi^{(k)}$ has only finitely many zeros in $[0,1]$. Further, let

$$
\psi(t):=\int_{-\infty}^{t} \varphi(\xi) \mathrm{d} \xi, \quad t \in \mathbb{R}
$$

then $\psi^{\prime}(t)=\varphi(t), t \in \mathbb{R}$. We study the boundedness of the "change of variable" operator

$$
T_{d}^{\psi}: f(x) \mapsto\left(\prod_{i=1}^{d} \psi^{\prime}\left(x_{i}\right)\right) \cdot f\left(\psi\left(x_{1}\right), \ldots, \psi\left(x_{d}\right)\right), \quad f \in L_{1}\left(\mathbb{R}^{d}\right), x \in \mathbb{R}^{d},
$$

in the situations

$$
T_{d}^{\psi}: \mathbf{B}_{p, \theta}^{s}\left(\mathbb{R}^{d}\right) \rightarrow \mathbf{B}_{p, \theta}^{s}\left(\mathbb{R}^{d}\right) \quad \text { and } \quad T_{d}^{\psi}: \mathbf{F}_{p, \theta}^{s}\left(\mathbb{R}^{d}\right) \rightarrow \mathbf{F}_{p, \theta}^{s}\left(\mathbb{R}^{d}\right)
$$

The following theorems represent the main results of the paper.

Theorem 4.1 Let $1 \leq p \leq \infty, 0<\theta \leq \infty, s>0$, and $\varphi \in C_{0}^{k}(\mathbb{R})$ as above, with $k>\lfloor s\rfloor+1$ if $p>1$ and $k>\lfloor s\rfloor+2$ if $p=1$. Then

$$
\left\|T_{d}^{\psi} f\right\|_{\mathbf{B}_{p, \theta}^{s}} \lesssim \psi\|f\|_{\mathbf{B}_{p, \theta}^{s}}, \quad f \in \mathbf{B}_{p, \theta}^{s}\left(\mathbb{R}^{d}\right)
$$

Theorem 4.2 Let $1<p<\infty, 1<\theta \leq \infty$, $s>0$, and $\varphi \in C_{0}^{k}(\mathbb{R})$ as above, with $k>\lfloor s\rfloor+1$. Then

$$
\left\|T_{d}^{\psi} f\right\|_{\mathbf{F}_{p, \theta}^{s}} \lesssim \psi\|f\|_{\mathbf{F}_{p, \theta}^{s}}, \quad f \in \mathbf{F}_{p, \theta}^{s}\left(\mathbb{R}^{d}\right)
$$

Remark 4.3 (i) Observe that the smoothness $k$ of kernel $\varphi$ in the F-case does not have to grow to infinity when $p$ tends to 1 . This result has to be compared with the mentioned result of Temlyakov, see (1.11) and [38, page 237].

(ii) With the technique used in this paper, we were not able to give a result in the case $p<1$ for both $\mathbf{B}$ and $\mathbf{F}$-spaces, not even in the univariate situation, see Lemma 4.4 below. Note that also the case $p=1$ is open in the $\mathbf{F}$-case.

For the convenience of the reader, we will first comment on a corresponding univariate result.

Lemma 4.4 Let $1<p<\infty, 1<\theta \leq \infty$, $s>0$ and $\varphi \in C_{0}^{k}(\mathbb{R})$ as above with $k>\lfloor s\rfloor+1$. Then

$$
\left\|T_{1}^{\psi} f\right\|_{F_{p, \theta}^{s}} \lesssim \psi\|f\|_{F_{p, \theta}^{s}}, \quad f \in F_{p, \theta}^{s}(\mathbb{R}) .
$$


Before we come to the proof of the univariate boundedness results, we need some preparation. It will turn out that difference characterizations of the function spaces under consideration is a suitable approach to analyze the respective operators.

\subsection{Proof of Lemma 4.4}

Let us first prove a technical lemma on the boundedness of quotients of derivatives of $\varphi$. Such terms naturally appear if one bounds the $L_{p}$-norm of $T_{1}^{\psi} f$. For the particular choice $\phi=\psi_{k}^{\prime}$, with $\psi_{k}$ from (1.4), the lemma below was proved in [36, p. 238].

Lemma 4.5 Let $1<p \leq \infty$ and $m \in \mathbb{N}_{0}$. Further, let $\phi \in C_{0}^{k}(\mathbb{R})$ with $\operatorname{supp}(\varphi) \subset$ $[0,1], \varphi>0$ on $(0,1)$ and $k>\frac{m p}{p-1}+1$. Let us further assume that the $k$ th derivative $\varphi^{(k)}$ has only finitely many zeros in $[0,1]$. Then we have

$$
\frac{\left|\varphi^{(n)}(t)\right|}{|\varphi(t)|^{1 / p}} \in L_{\infty}([0,1])
$$

for all $0 \leq n \leq m$.

Proof It is enough to prove the result for $n=m$. If $p=\infty$, the result is obvious. Hence, assume $1<p<\infty$. We put $\ell=k-1$. Using Taylor's theorem and the fact that $\phi^{(i)}(0)=0$ for all $i=0, \ldots, k$, we obtain

$$
\phi(t)=\frac{1}{\ell !} \int_{0}^{t} \phi^{(\ell+1)}(\xi)(t-\xi)^{\ell} \mathrm{d} \xi
$$

as well as

$$
\phi^{(m)}(t)=\frac{1}{(\ell-m) !} \int_{0}^{t} \phi^{(\ell+1)}(\xi)(t-\xi)^{\ell-m} \mathrm{~d} \xi
$$

for all $t \in[0,1]$. Since $\varphi^{(\ell+1)}$ has only finitely many zeros in $[0,1]$, there exists an $\varepsilon>0$ such that $\phi^{(\ell+1)}(t)>0$ for $t \in(0, \varepsilon)$. This shows with $p^{\prime}=p /(p-1)$ that

$$
\begin{aligned}
\frac{\left|\phi^{(m)}(t)\right|}{|\phi(t)|^{1 / p}} & \leq \frac{\ell !}{(\ell-m) !} \frac{t^{1 / p^{\prime}}\left(\int_{0}^{t}\left(\phi^{(\ell+1)}(\xi)\right)^{p}(t-\xi)^{p(\ell-m)} \mathrm{d} \xi\right)^{1 / p}}{\left(\int_{0}^{t} \phi^{(\ell+1)}(\xi)(t-\xi)^{\ell} \mathrm{d} \xi\right)^{1 / p}} \\
& \lesssim_{k} \sup _{\xi \in(0, \varepsilon)}\left(\left|\phi^{(\ell+1)}(\xi)\right|^{p-1}|t-\xi|^{(\ell-m)-\ell}\right)^{1 / p} \frac{\left(\int_{0}^{t} \phi^{(\ell+1)}(\xi)(t-\xi)^{\ell} \mathrm{d} \xi\right)^{1 / p}}{\left(\int_{0}^{t} \phi^{(\ell+1)}(\xi)(t-\xi)^{\ell} \mathrm{d} \xi\right)^{1 / p}} \\
& \lesssim_{k} 1
\end{aligned}
$$

for $t \in(0, \varepsilon)$. In the last inequality, we used $p>1$ and $\ell>\frac{p m}{p-1}$. The same arguments work also for $\left(1-\varepsilon_{0}, 1\right)$ with some $\varepsilon_{0}>0$. The quotient is uniformly bounded in $\left[\varepsilon, 1-\varepsilon_{0}\right]$ since $\phi(t) \geq c>0$ for $t \in\left[\varepsilon, 1-\varepsilon_{0}\right]$. 
It is easily seen that Lemma 4.5 is not true for $p=1$. We immediately obtain the following corollary. Note the relaxed conditions on $p$ and $k$.

Corollary 4.6 Let $1 \leq p \leq \infty$ and $m \in \mathbb{N}_{0}$. Further, let $\phi \in C_{0}^{k}(\mathbb{R})$ with $\operatorname{supp}(\varphi) \subset$ $[0,1], \varphi>0$ on $(0,1)$, and $k>m+1$. Let us further assume that the $k$ th derivative $\varphi^{(k)}$ has only finitely many zeros in $[0,1]$. Then we have

$$
\frac{\left|\varphi^{(r)}(t) \varphi^{(\alpha)}(t)\right|}{|\varphi(t)|^{1 / p}} \in L_{\infty}([0,1])
$$

for all $0 \leq r, \alpha \leq m$ with $r+\alpha \leq m$.

Proof Obviously, it is enough to prove the statement for $p=1$, since

$$
\frac{\left|\varphi^{(r)}(t) \varphi^{(\alpha)}(t)\right|}{|\varphi(t)|^{1 / p}} \lesssim \frac{\left|\varphi^{(r)}(t) \varphi^{(\alpha)}(t)\right|}{|\varphi(t)|}
$$

due to $\varphi \in C_{0}^{k}(\mathbb{R})$. We write

$$
\frac{\left|\varphi^{(r)}(t) \varphi^{(\alpha)}(t)\right|}{|\varphi(t)|}=\frac{\left|\varphi^{(r)}(t)\right|}{|\varphi(t)|^{1 / p_{1}}} \cdot \frac{\left|\varphi^{(\alpha)}(t)\right|}{|\varphi(t)|^{1 / p_{2}}}
$$

for some $p_{1}, p_{2}$ with $1 / p_{1}+1 / p_{2}=1$. Using Lemma 4.5 , we know that we need $k>\frac{r p_{1}}{p_{1}-1}+1$ for the boundedness of the first factor and $k>\frac{\alpha p_{2}}{p_{2}-1}+1$ for the boundedness of the second.

First note that the statement of the corollary is trivial for $r=\alpha=0$. If only one of them is zero, say $r=0$, then we set $p_{1}=1$ and $p_{2}=\infty$; again the statement is obvious. Hence, we assume now that $r \neq 0$ and $\alpha \neq 0$, and we choose $p_{1}=(r+\alpha) / \alpha$ and $p_{2}=(r+\alpha) / r$.

This leads to the restriction $k>r+\alpha+1$ in both cases. Since we want a result for all $r+\alpha \leq m$, we impose the restriction $k>m+1$. This finishes the proof.

Now we are in position to prove Lemma 4.4.

Proof of Lemma 4.4. Step 1 Let $\eta_{j}$ denote a smooth dyadic compactly supported decomposition of unity (for instance the one given at the beginning of Sect. 3.1). In particular, $1=\sum_{j \in \mathbb{N}_{0}} \eta_{j}(t)$ for all $t \in \mathbb{R}$, and $\eta_{j}(t)=0$ if $|t|>2^{j+1}$. We decompose $f$ as follows:

$$
f(t)=\sum_{j \in \mathbb{Z}} f_{j}(t):=\sum_{j \in \mathbb{Z}} \mathcal{F}^{-1}\left[\eta_{j} \mathcal{F} f\right](t),
$$

where we put $f_{j} \equiv 0$ in case $j<0$. Note that with this definition, we can clearly also write $f=\sum_{\ell \in \mathbb{Z}} f_{j+\ell}$ for every fixed $j \in \mathbb{N}_{0}$. Using the characterization by rectangular means of differences of $F_{p, \theta}^{s}$, see Theorem 3.7, and the decomposition (4.1), we have 


$$
\begin{aligned}
\left\|T_{1}^{\psi} f\right\|_{F_{p, \theta}^{s}}^{(m)} \leq & \sum_{\ell \in \mathbb{Z}}\left\|\left(\sum_{j \in \mathbb{N}_{0}} 2^{j s \theta}\left[2^{j} \int_{-2^{-j}}^{2^{-j}}\left|\Delta_{h}^{m}\left(T_{1}^{\psi} f_{j+\ell}, \cdot\right)\right| \mathrm{d} h\right]^{\theta}\right)^{1 / \theta}\right\|_{p} \\
= & \sum_{\ell<0}\left\|\left(\sum_{j \in \mathbb{N}_{0}} 2^{j s \theta}\left[2^{j} \int_{-2^{-j}}^{2^{-j}}\left|\Delta_{h}^{m}\left(T_{1}^{\psi} f_{j+\ell}, \cdot\right)\right| \mathrm{d} h\right]^{\theta}\right)^{1 / \theta}\right\|_{p} \\
& +\sum_{\ell \geq 0}\left\|\left(\sum_{j \in \mathbb{N}_{0}} 2^{j s \theta}\left[2^{j} \int_{-2^{-j}}^{2^{-j}}\left|\Delta_{h}^{m}\left(T_{1}^{\psi} f_{j+\ell}, \cdot\right)\right| \mathrm{d} h\right]^{\theta}\right)^{1 / \theta}\right\|_{p} .
\end{aligned}
$$

Here we choose $m=\lfloor s\rfloor+1$.

Step 2 First we recall the convolution representation of the $m$ th order difference. Let $B_{m}(\cdot)$ be a univariate B-spline of degree $m$ which has knots at the points $\{0,1, \ldots, m\}$; i.e.,

$$
B_{m}=\chi_{[0,1]} * \ldots * \chi_{[0,1]}, \quad m \text { times, }
$$

where $\chi_{[0,1]}$ denotes the characteristic function of $[0,1]$. Note that $B_{m}(\cdot)$ is bounded and $\operatorname{supp}\left(B_{m}\right) \subset[0, m]$. In case $h>0$, we have

$$
\Delta_{h}^{m}\left(T_{1}^{\psi} f_{j+\ell}, t\right)=h^{m-1} \int_{0}^{h m}\left(T_{1}^{\psi} f_{j+\ell}\right)^{(m)}(t+\xi) B_{m}\left(h^{-1} \xi\right) \mathrm{d} \xi,
$$

see [5, page 45]. If $h<0$, we use B-spline with knots $\{-m, \ldots, 0\}$ and get a similar formula. Consequently, we obtain for $|h|<2^{-j}$,

$$
\begin{aligned}
\left|\Delta_{h}^{m}\left(T_{1}^{\psi} f_{j+\ell}, t\right)\right| & \lesssim|h|^{m} M\left[\left(T_{1}^{\psi} f_{j+\ell}\right)^{(m)}\right](t) \\
& \leq 2^{-j m} M\left[\left(T_{1}^{\psi} f_{j+\ell}\right)^{(m)}\right](t)
\end{aligned}
$$

since $B_{m}\left(h^{-1} \cdot\right)$ is bounded. This leads to

$$
\begin{aligned}
\sum_{\ell<0} \| & \left(\sum_{j \in \mathbb{N}_{0}} 2^{j s \theta}\left[2^{j} \int_{-2^{-j}}^{2^{-j}}\left|\Delta_{h}^{m}\left(T_{1}^{\psi} f_{j+\ell}, \cdot\right)\right| \mathrm{d} h\right]^{\theta}\right)^{1 / \theta} \|_{p} \\
& \lesssim \sum_{\ell<0}\left\|\left(\sum_{j \in \mathbb{N}_{0}} 2^{(s-m) j \theta}\left(M\left[\left(T_{1}^{\psi} f_{j+\ell}\right)^{(m)}\right](\cdot)\right)^{\theta}\right)^{1 / \theta}\right\|_{p} \\
& \lesssim \sum_{\ell<0}\left\|\left(\sum_{j \in \mathbb{N}_{0}} 2^{(s-m) j \theta}\left|\left(T_{1}^{\psi} f_{j+\ell}\right)^{(m)}(\cdot)\right|^{\theta}\right)^{1 / \theta}\right\|_{p}
\end{aligned}
$$

where in the second inequality we have applied Theorem 2.1. Now we continue with

$$
\left(T_{1}^{\psi} f_{j+\ell}\right)^{(m)}(t)=\left[\varphi f_{j+\ell}(\psi)\right]^{(m)}(t)=\sum_{\alpha=0}^{m} C_{\alpha} \varphi^{(\alpha)}(t)\left[f_{j+\ell}(\psi)\right]^{(m-\alpha)}(t)
$$


for some $C_{\alpha}$. We distinguish two cases. First let us deal with $\alpha=m$. We choose the number $a$ such that $\max \{1 / p, 1 / \theta\}<a \leq m$. This is possible since $\max \{1 / p, 1 / \theta\}<$ 1 and $m \geq 1$. Then, if $t \in[0,1]$, we have

$$
\begin{aligned}
\left|\varphi^{(m)}(t) f_{j+\ell}(\psi(t))\right| & \lesssim \sup _{\xi \in[0,1]}\left|f_{j+\ell}(\xi)\right| \\
& =\sup _{\xi \in[0,1]} \frac{\left|f_{j+\ell}(\xi)\right|}{\left(1+2^{j+\ell}|t-\xi|\right)^{a}}\left(1+2^{j+\ell}|t-\xi|\right)^{a} \\
& \lesssim 2^{(\ell+j) a} \sup _{\xi \in[0,1]} \frac{\left|f_{j+\ell}(\xi)\right|}{\left(1+2^{j+\ell}|t-\xi|\right)^{a}} \\
& \lesssim 2^{(\ell+j) m} \sup _{\xi \in \mathbb{R}} \frac{\left|f_{j+\ell}(\xi)\right|}{\left(1+2^{j+\ell}|t-\xi|\right)^{a}} \\
& =2^{(\ell+j) m} P_{j+\ell, a}\left(f_{j+\ell}, t\right) .
\end{aligned}
$$

If $t \notin[0,1]$, the inequality (4.4) is obviously satisfied since $\operatorname{supp}(\varphi) \subset[0,1]$. We consider the summand

$$
\begin{aligned}
& \left\|\left(\sum_{j \in \mathbb{N}_{0}} 2^{(s-m) j \theta}\left|\varphi^{(m)}(\cdot) f_{j+\ell}(\psi(\cdot))\right|^{\theta}\right)^{1 / \theta}\right\|_{p} \\
& \lesssim 2^{(m-s) \ell}\left\|\left(\sum_{j \in \mathbb{N}_{0}} 2^{(\ell+j) s \theta} P_{j+\ell, a}\left(f_{j+\ell}, \cdot\right)^{\theta}\right)^{1 / \theta}\right\|_{p} \lesssim 2^{(m-s) \ell}\|f\|_{F_{p, \theta}^{s}} .
\end{aligned}
$$

In the last step, we used Theorem 2.4. In case $\alpha<m$, we use Faà di Bruno's formula for the chain rules of higher derivatives to obtain

$$
\varphi^{(\alpha)}(t)\left[f_{j+\ell}(\psi)\right]^{(m-\alpha)}(t)=\left.\sum_{1 \leq \gamma \leq m-\alpha} f_{j+\ell}^{(\gamma)}(\xi)\right|_{\xi=\psi(t)} \cdot \varphi^{(\alpha)}(t) \cdot Q_{\gamma}(t)
$$

where $Q_{\gamma}(t)$ has the form

$$
Q_{\gamma}(t)=\sum_{k_{1}, \ldots, k_{m-\alpha}} \frac{(m-\alpha) !}{k_{1} ! k_{2} ! \ldots k_{m-\alpha} !}\left(\frac{\varphi(t)}{1 !}\right)^{k_{1}}\left(\frac{\varphi^{\prime}(t)}{2 !}\right)^{k_{2}} \cdots\left(\frac{\varphi^{(m-\alpha-1)}(t)}{(m-\alpha) !}\right)^{k_{m-\alpha}}
$$

with the sum taken over all nonnegative integer $k_{1}, \ldots, k_{m-\alpha}$ such that

$$
k_{1}+2 k_{2}+\ldots+(m-\alpha) k_{m-\alpha}=m-\alpha \text { and } k_{1}+k_{2}+\ldots+k_{m-\alpha}=\gamma \text {. }
$$

Since the highest order derivative of $\varphi(t)$ in $Q_{\gamma}(t)$ is $m-\alpha-1$ and $\varphi \in C_{0}^{k}(\mathbb{R})$, $k>\lfloor s\rfloor+1=m$, Corollary 4.6 yields that

$$
\frac{\left|\varphi^{(r)}(t) \varphi^{(\alpha)}(t)\right|}{|\varphi(t)|^{1 / p}}<C
$$


for all $r, \alpha$ with $r+\alpha \leq m-1$, which leads to $\left|\varphi^{(\alpha)}(t) Q_{\gamma}(t)\right| \lesssim|\varphi(t)|^{1 / p}$. Consequently,

$$
\left|\varphi^{(\alpha)}(t)\left[f_{j+\ell}(\psi)\right]^{(m-\alpha)}(t)\right| \lesssim \sum_{1 \leq \gamma \leq m-\alpha}\left|f_{j+\ell}^{(\gamma)}(\xi)\right|_{\xi=\psi(t)} \cdot|\varphi(t)|^{1 / p} .
$$

We consider the term

$$
\begin{aligned}
F_{\alpha, \ell} & :=\left\|\left(\sum_{j \in \mathbb{N}_{0}} 2^{(s-m) j \theta}\left|\varphi^{(\alpha)}\left[f_{j+\ell}(\psi)\right]^{(m-\alpha)}\right|^{\theta}\right)^{1 / \theta}\right\|_{p} \\
& \lesssim \sum_{1 \leq \gamma \leq m-\alpha}\left\|\left(\left.\sum_{j \in \mathbb{N}_{0}} 2^{(s-m) j \theta}\left|f_{j+\ell}^{(\gamma)}(\xi)\right|_{\xi=\psi(\cdot)} \cdot \varphi^{1 / p}\right|^{\theta}\right)^{1 / \theta}\right\|_{p} \\
& \lesssim \sum_{1 \leq \gamma \leq m-\alpha}\left\|\left(\sum_{j \in \mathbb{N}_{0}} 2^{(s-m) j \theta}\left|f_{j+\ell}^{(\gamma)}\right|^{\theta}\right)^{1 / \theta}\right\|_{p} \cdot
\end{aligned}
$$

By Lemma 2.3 and a homogeneity argument, we have for any $a>0$,

$$
\left|f_{j+\ell}^{(\gamma)}(t)\right| \leq \sup _{\xi \in \mathbb{R}} \frac{\left|f_{j+\ell}^{(\gamma)}(\xi)\right|}{\left(1+2^{j+\ell}|t-\xi|\right)^{a}} \lesssim 2^{(j+\ell) m} \sup _{\xi \in \mathbb{R}} \frac{\left|f_{j+\ell}(\xi)\right|}{\left(1+2^{j+\ell}|t-\xi|\right)^{a}}
$$

if $1 \leq \gamma \leq m$. Putting this into (4.7) with $a>\max \{1 / p, 1 / \theta\}$ and using Theorem 2.4 afterwards yields

$$
\begin{aligned}
F_{\alpha, \ell} & \lesssim \sum_{1 \leq \gamma \leq m-\alpha} 2^{\ell(m-s)}\left\|\left(\sum_{j \in \mathbb{N}_{0}} 2^{(j+\ell) s \theta}\left|P_{j+\ell, a}\left(f_{j+\ell}, \cdot\right)\right|^{\theta}\right)^{1 / \theta}\right\|_{p} \\
& \lesssim 2^{\ell(m-s)}\|f\|_{F_{p, \theta}^{s}} .
\end{aligned}
$$

From (4.5), (4.7), and (4.9) we obtain

$$
\begin{aligned}
\sum_{\ell<0} \|\left(\sum _ { j \in \mathbb { N } _ { 0 } } 2 ^ { j s \theta } \left[2^{j} \int_{-2^{-j}}^{2^{-j}} \mid \Delta_{h}^{m}\right.\right. & \left.\left.\left(T_{1}^{\psi} f_{j+\ell}, \cdot\right) \mid \mathrm{d} h\right]^{\theta}\right)^{1 / \theta} \|_{p} \\
& \lesssim \sum_{\ell<0} 2^{\ell(m-s)}\|f\|_{F_{p, \theta}^{s}} \lesssim\|f\|_{F_{p, \theta}^{s}}
\end{aligned}
$$

Step 3 It remains to deal with $\sum_{\ell \geq 0}$. We expand the difference and estimate

$$
\begin{aligned}
\sum_{\ell \geq 0} \| & \left(\sum_{j \in \mathbb{N}_{0}} 2^{j s \theta}\left[2^{j} \int_{-2^{-j}}^{2^{-j}}\left|\Delta_{h}^{m}\left(T_{1}^{\psi} f_{j+\ell}, \cdot\right)\right| \mathrm{d} h\right]^{\theta}\right)^{1 / \theta} \|_{p} \\
& \lesssim \sum_{\ell \geq 0} \sum_{i=0}^{m}\left\|\left(\sum_{j \in \mathbb{N}_{0}} 2^{j s \theta}\left[2^{j} \int_{-2^{-j}}^{2^{-j}}\left|T_{1}^{\psi} f_{j+\ell}(\cdot+i h)\right| \mathrm{d} h\right]^{\theta}\right)^{1 / \theta}\right\|_{p} .
\end{aligned}
$$


We consider the term

$$
\begin{aligned}
A_{i, \ell} & =\left\|\left(\sum_{j \in \mathbb{N}_{0}} 2^{j s \theta}\left[2^{j} \int_{-2^{-j}}^{2^{-j}}\left|\left(T_{1}^{\psi} f_{j+\ell}\right)(\cdot+i h)\right| \mathrm{d} h\right]^{\theta}\right)^{1 / \theta}\right\|_{p} \\
& \lesssim\left\|\left(\sum_{j \in \mathbb{N}_{0}} 2^{j s \theta}\left(M\left[T_{1}^{\psi} f_{j+\ell}\right](\cdot)\right)^{\theta}\right)^{1 / \theta}\right\|_{p} .
\end{aligned}
$$

Here again we use the Hardy-Littlewood maximal function. From Theorem 2.1 again, we get

$$
\begin{aligned}
A_{i, \ell} & \lesssim\left\|\left(\sum_{j \in \mathbb{N}_{0}} 2^{j s \theta}\left|\left(T_{1}^{\psi} f_{j+\ell}\right)\right|^{\theta}\right)^{1 / \theta}\right\|_{p} \\
& \lesssim 2^{-\ell s}\left\|\left(\sum_{j \in \mathbb{N}_{0}} 2^{(j+\ell) s \theta}\left|\varphi(\cdot) f_{j+\ell}(\psi(\cdot))\right|^{\theta}\right)^{1 / \theta}\right\|_{p} \\
& \lesssim 2^{-\ell s}\left\|\left(\sum_{j \in \mathbb{N}_{0}} 2^{(j+\ell) s \theta}\left|f_{j+\ell}\right|^{\theta}\right)^{1 / \theta}\right\|_{p} \\
& \lesssim 2^{-\ell s}\|f\|_{F_{p, \theta}^{s}} .
\end{aligned}
$$

We performed a change of variable in the second step and used $p>1$ and $\phi \in C_{0}^{k}(\mathbb{R})$. With (4.11), (4.12), and (4.13), we obtain

$$
\begin{aligned}
\sum_{\ell \geq 0} \|\left(\sum_{j \in \mathbb{N}_{0}} 2^{j s \theta}\right. & {\left.\left[2^{j} \int_{-2^{-j}}^{2^{-j}}\left|\Delta_{h}^{m}\left(T_{1}^{\psi} f_{j+\ell}, \cdot\right)\right| \mathrm{d} h\right]^{\theta}\right)^{1 / \theta} \|_{p} } \\
& \lesssim \sum_{i=1}^{m} \sum_{\ell \geq 0} A_{i, \ell} \lesssim \sum_{\ell \geq 0} 2^{-\ell s}\|f\|_{F_{p, \theta}^{s}} \lesssim\|f\|_{F_{p, \theta}^{s}},
\end{aligned}
$$

where we used $s>0$ in the last inequality. Altogether we obtain from (4.10) and (4.14),

$$
\left\|\left(\sum_{j \in \mathbb{N}_{0}} 2^{j s \theta}\left[2^{j} \int_{-2^{-j}}^{2^{-j}}\left|\Delta_{h}^{m}\left(T_{1}^{\psi} f, \cdot\right)\right| \mathrm{d} h\right]^{\theta}\right)^{1 / \theta}\right\|_{p} \lesssim\|f\|_{F_{p, \theta}^{s}}
$$

In view of (4.2), we conclude that

$$
\left\|T_{1}^{\psi} f\right\|_{F_{p, \theta}^{s}}^{(m)} \lesssim\|f\|_{F_{p, \theta}^{s}},
$$

which finishes the proof. 


\subsection{Proof of Theorems 4.1 and 4.2}

Proof of Theorem 4.2. Step 1 We aim at adapting the proof of Lemma 4.4 to the multivariate mixed situation by applying the arguments direction-wise. The proper tool will be the characterization by rectangular means of differences, see Theorem 3.7. Let $\left\{\eta_{j}\right\}_{j \in \mathbb{N}_{0}^{d}}$ be a tensorized compactly supported smooth dyadic decomposition of unity and

$$
f_{j}:=\mathcal{F}^{-1}\left[\eta_{j} \mathcal{F} f\right] \quad, \quad j \in \mathbb{Z}^{d},
$$

where we put $f_{j} \equiv 0$ if $j \notin \mathbb{N}_{0}^{d}$. Analogously to (4.2), we estimate the quantity

$$
\begin{gathered}
\left\|\left(\sum_{j \in \mathbb{N}_{0}^{d}} 2^{|j|_{1} s \theta} \mathcal{R}_{m}^{e(j)}\left(T_{d}^{\psi} f, 2^{-j}, \cdot\right)^{\theta}\right)^{1 / \theta}\right\|_{p} \\
\leq \sum_{\ell \in \mathbb{Z}^{d}}\left\|\left(\sum_{j \in \mathbb{N}_{0}^{d}} 2^{|j|_{1} s \theta} \mathcal{R}_{m}^{e(j)}\left(T_{d}^{\psi} f_{j+\ell}, 2^{-j}, \cdot\right)^{\theta}\right)^{1 / \theta}\right\|_{p},
\end{gathered}
$$

where $2^{-j}=\left(2^{-j_{1}}, \ldots, 2^{-j_{d}}\right)$. Again we choose $m=\lfloor s\rfloor+1$. Let us introduce some further notation first. If $e \subset[d]$ and $j \in \mathbb{N}_{0}^{d}$, we write

$$
\Gamma_{j}^{e}=\underset{i \in e}{X}\left[-2^{-j_{i}}, 2^{-j_{i}}\right]
$$

For each $\ell \in \mathbb{Z}^{d}$, we write

$$
e_{1}(j, \ell)=e(j) \cap\left\{i: \ell_{i} \geq 0\right\} \quad \text { and } \quad e_{2}(j, \ell)=e(j) \cap\left\{i: \ell_{i}<0\right\} .
$$

For simplicity, we put

$$
F_{\ell}:=\left\|\left(\sum_{j \in \mathbb{N}_{0}^{d}} 2^{|j|_{1} s \theta} \mathcal{R}_{m}^{e(j)}\left(T_{d}^{\psi} f_{j+\ell}, 2^{-j}, \cdot\right)^{\theta}\right)^{1 / \theta}\right\|_{p} .
$$

Step 2 Estimation of $F_{\ell}$. We have

$$
\begin{aligned}
\Delta_{h}^{m, e(j)}\left(T_{d}^{\psi} f_{j+\ell}, x\right) & =\Delta_{h}^{m, e_{1}(j, \ell)} \circ \Delta_{h}^{m, e_{2}(j, \ell)}\left(T_{d}^{\psi} f_{j+\ell}, x\right) \\
& =\sum_{k} \Delta_{h}^{m, e_{2}(j, \ell)}\left(T_{d}^{\psi} f_{j+\ell}, x+(k \cdot h)\right),
\end{aligned}
$$

where the sum on the right-hand side is taken over all $k \in \mathbb{N}_{0}^{d}$ such that $0 \leq k_{i} \leq m$ if $i \in e_{1}(j, \ell)$ and $k_{i}=0$ otherwise. In addition, we put $(k \cdot h)=\left(k_{1} h_{1}, \ldots, k_{d} h_{d}\right)$. Using the convolution representation of the $m$ th order difference in $e_{2}(j, \ell)$, we have the inequality 


$$
\begin{aligned}
\mid \Delta_{h}^{m, e_{2}(j, \ell)} & \left(T_{d}^{\psi} f_{j+\ell}, x+(k \cdot h)\right) \mid \\
& \lesssim\left(\prod_{i \in e_{2}(j, \ell)} 2^{-j_{i} m}\right) M_{e_{2}(j, \ell)}\left[D^{\left(m_{e_{2}(j, \ell)}\right)}\left(T_{d}^{\psi} f_{j+\ell}\right)\right](x+(k \cdot h)),
\end{aligned}
$$

see (4.3). Here $m_{e_{2}(j, \ell)}=\left(m_{1}, \ldots, m_{d}\right)$, where $m_{i}=m$ if $i \in e_{2}(j, \ell)$, and otherwise $m_{i}=0$. This leads to

$$
\begin{aligned}
& \mathcal{R}_{m}^{e(j)}\left(T_{d}^{\psi} f_{j+\ell}, 2^{-j}, x\right) \\
& \quad \lesssim \sum_{k} \int_{\Gamma_{j}^{e(j)}}\left|\Delta_{h}^{m, e_{2}(j, \ell)}\left(T_{d}^{\psi} f_{j+\ell}, x+(k \cdot h)\right)\right| \prod_{i \in e(j)} 2^{j_{i}} \mathrm{~d} h_{i} \\
& \quad \lesssim \sum_{k} \int_{\Gamma_{j}^{e(j)}}\left(\prod_{i \in e_{2}(j, \ell)} 2^{-j_{i} m}\right) M_{e_{2}(j, \ell)}\left[D^{\left(m_{e_{2}(j, \ell)}\right)}\left(T_{d}^{\psi} f_{j+\ell}\right)\right](x+(k \cdot h)) \prod_{i \in e(j)} 2^{j_{i}} \mathrm{~d} h_{i} .
\end{aligned}
$$

Now we estimate above by the Hardy-Littlewood maximal function with the components in $e_{1}(j, \ell)$ and obtain

$$
\begin{aligned}
\mathcal{R}_{m}^{e(j)}\left(T_{d}^{\psi} f_{j+\ell}, 2^{-j}, x\right) & \lesssim\left(\prod_{i \in e_{2}(j, \ell)} 2^{-j_{i} m}\right) M_{e_{1}(j, \ell)}\left[M_{e_{2}(j, \ell)}\left[D^{\left(m_{e_{2}(j, \ell)}\right)}\left(T_{d}^{\psi} f_{j+\ell}\right)\right]\right](x) \\
& \lesssim\left(\prod_{i \in e_{2}(j, \ell)} 2^{-j_{i} m}\right) M_{[d]}\left[D^{\left(m_{e_{2}(j, \ell)}\right)}\left(T_{d}^{\psi} f_{j+\ell}\right)\right](x) .
\end{aligned}
$$

Plugging this into $F_{\ell}$, Theorem 2.1 yields

$$
F_{\ell} \lesssim\left\|\left(\sum_{j \in \mathbb{N}_{0}^{d}} 2^{|j|_{1} s \theta}\left(\prod_{i \in e_{2}(j, \ell)} 2^{-j_{i} m \theta}\right)\left|D^{\left(m_{e_{2}(j, \ell)}\right)}\left(T_{d}^{\psi} f_{j+\ell}\right)(\cdot)\right|^{\theta}\right)^{1 / \theta}\right\|_{p}
$$

Using Leibniz's formula, we estimate

$$
\begin{aligned}
\left|D^{\left(m_{e_{2}(j, \ell)}\right)}\left(T_{d}^{\psi} f_{j+\ell}\right)(x)\right| & =\left|D^{\left(m_{e_{2}(j, \ell)}\right)}\left(\left[\bigotimes_{i=1}^{d} \varphi\right] f_{j+\ell}(\psi, \ldots, \psi)\right)(x)\right| \\
& \lesssim \sum_{\alpha}\left|D^{\alpha}\left[\bigotimes_{i=1}^{d} \varphi\right](x) D^{\beta}\left[f_{j+\ell}(\psi, \ldots, \psi)\right](x)\right|
\end{aligned}
$$

Here the sum on the right-hand side is taken over all $\alpha \in \mathbb{N}_{0}^{d}$ with $0 \leq \alpha_{i} \leq m$ if $i \in e_{2}(j, \ell)$; otherwise $\alpha_{i}=0$ and $\beta=m_{e_{2}(j, \ell)}-\alpha$. We continue estimation by dividing $e_{2}(j, \ell)=e_{2}^{1}(j, \ell) \cup e_{2}^{2}(j, \ell)$, where $\alpha_{i}<m$ if $i \in e_{2}^{1}(j, \ell)$ and $\alpha_{i}=m$ if $i \in e_{2}^{2}(j, \ell)$. We have 


$$
\begin{aligned}
D^{\alpha} & {\left[\bigotimes_{i=1}^{d} \varphi\right](x) D^{\beta}\left[f_{j+\ell}(\psi, \ldots, \psi)\right](x) } \\
= & \left(\prod_{i \in e_{0}(j) \cup e_{1}(j, \ell)} \varphi\left(x_{i}\right)\right)\left(\prod_{i \in e_{2}^{2}(j, \ell)} \varphi^{(m)}\left(x_{i}\right)\right)\left(\prod_{i \in e_{2}^{1}(j, \ell)} \varphi^{\left(\alpha_{i}\right)}\left(x_{i}\right)\right) \\
& \times D^{\beta}\left[f_{j+\ell}(\psi, \ldots, \psi)\right](x) .
\end{aligned}
$$

Now the condition $\varphi \in C_{0}^{k}(\mathbb{R}), k>m$, implies that

$$
\begin{aligned}
\mid\left(\prod_{i \in e_{2}^{1}(j, \ell)} \varphi^{\left(\alpha_{i}\right)}\left(x_{i}\right)\right) & D^{\beta}\left[f_{j+\ell}(\psi, \ldots, \psi)\right](x) \mid \\
& \lesssim \sum_{\gamma}\left(\prod_{i \in e_{2}^{1}(j, \ell)}\left|\varphi\left(x_{i}\right)\right|^{1 / p}\right) \cdot \mid\left[D^{(\gamma)} f_{j+\ell}\right]\left(\psi\left(x_{1}\right), \ldots, \psi\left(x_{d}\right) \mid,\right.
\end{aligned}
$$

where the sum is taken over all $\gamma \in \mathbb{N}_{0}^{d}$ such that $1 \leq \gamma_{i} \leq \beta_{i}$ if $i \in e_{2}^{1}(j, \ell)$ and $\gamma_{i}=0$ otherwise, see (4.6). This leads to

$$
\begin{aligned}
& \left|D^{\alpha}\left[\bigotimes_{i=1}^{d} \varphi\right](x) D^{\beta}\left[f_{j+\ell}(\psi, \ldots, \psi)\right](x)\right| \\
& \lesssim \sum_{\gamma}\left(\prod_{i \in[d] \backslash e_{2}^{2}(j, \ell)}\left|\varphi\left(x_{i}\right)\right|^{1 / p}\right) \cdot\left|\left(\prod_{i \in e_{2}^{2}(j, \ell)} \varphi^{(m)}\left(x_{i}\right)\right)\left[D^{\gamma} f_{j+\ell}\right]\left(\psi\left(x_{1}\right), \ldots, \psi\left(x_{d}\right)\right)\right| .
\end{aligned}
$$

Changing variable, we obtain

$$
\begin{aligned}
& \left\|\left(\sum_{j \in \mathbb{N}_{0}^{d}} 2^{|j|_{1} s \theta}\left(\prod_{i \in e_{2}(j, \ell)} 2^{-j_{i} m \theta}\right)\left|D^{\alpha}\left[\bigotimes_{i=1}^{d} \varphi\right] \cdot D^{\beta}\left[f_{j+\ell}(\psi, \ldots, \psi)\right]\right|^{\theta}\right)^{1 / \theta}\right\|_{p} \\
& \lesssim \sum_{\gamma}\left\|\left(\sum_{j \in \mathbb{N}_{0}^{d}} 2^{|j|_{1} s \theta}\left(\prod_{i \in e_{2}(j, \ell)} 2^{-j_{i} m \theta}\right)\left|\left(\prod_{i \in e_{2}^{2}(j, \ell)} \varphi^{(m)}\left(x_{i}\right)\right)\left[D^{\gamma} f_{j+\ell}\right]\left(x, \psi_{e_{2}^{2}(j, \ell)}\right)\right|^{\theta}\right)^{1 / \theta}\right\|_{p} .
\end{aligned}
$$

Here $\left[D^{\gamma} f_{j+\ell}\right]\left(x, \psi_{e_{2}^{2}(j, \ell)}\right)=\left[D^{\gamma} f_{j+\ell}\right]\left(z_{1}, \ldots, z_{d}\right)$ with $z_{i}=\psi\left(x_{i}\right)$ if $i \in e_{2}^{2}(j, \ell)$; otherwise $z_{i}=x_{i}$. Using the same scaling argument as in (4.4) and (4.8) yields 


$$
\begin{aligned}
& \left|\left(\prod_{i \in e_{2}^{2}(j, \ell)} \varphi^{(m)}\left(x_{i}\right)\right) D^{\gamma}\left[f_{j+\ell}\right]\left(x, \psi_{e_{2}^{2}(j, \ell)}\right)\right| \\
& \lesssim\left(\prod_{i \in e_{2}^{2}(j, \ell)} 2^{\left(j_{i}+\ell_{i}\right) m}\right) \sup _{y \in \mathbb{R}^{d}} \frac{\left|D^{\gamma} f_{j+\ell}(y)\right|}{\prod_{i=1}^{d}\left(1+2^{j_{i}+\ell_{i}}\left|x_{i}-y_{i}\right|\right)^{a}} \\
& \lesssim\left(\prod_{i \in e_{2}^{2}(j, \ell)} 2^{\left(j_{i}+\ell_{i}\right) m}\right)\left(\prod_{i \in e_{2}^{1}(j, \ell)} 2^{\left(j_{i}+\ell_{i}\right) \gamma_{i}}\right) \sup _{y \in \mathbb{R}^{d}} \frac{\left|f_{j+\ell}(y)\right|}{\prod_{i=1}^{d}\left(1+2^{j_{i}+\ell_{i}}\left|x_{i}-y_{i}\right|\right)^{a}} \\
& \lesssim\left(\prod_{i \in e_{2}(j, \ell)} 2^{\left(j_{i}+\ell_{i}\right) m}\right) P_{2^{j+\ell}, a} f_{j+\ell}(x),
\end{aligned}
$$

with $m \geq a$. Now we obtain from (4.20), (4.21), and (4.22),

$$
\begin{aligned}
F_{\ell} & \lesssim\left\|\left(\sum_{j \in \mathbb{N}_{0}^{d}} 2^{|j|_{1} s \theta}\left(\prod_{i \in e_{2}(j, \ell)} 2^{\ell_{i} m \theta}\right)\left|P_{2^{j+\ell}, a} f_{j+\ell}\right|^{\theta}\right)^{1 / \theta}\right\|_{p} \\
& \lesssim\left\|\left[\sum_{j \in \mathbb{N}_{0}^{d}}\left(\prod_{i \in e_{2}(j, \ell)} 2^{\ell_{i}(m-s) \theta}\right)\left(\prod_{i \in e_{0}(j) \cup e_{1}(j, \ell)} 2^{-s \ell_{i} \theta}\right) 2^{|j+\ell|_{1} s \theta}\left|P_{2^{j+\ell}, a} f_{j+\ell}\right|^{\theta}\right]^{1 / \theta}\right\|_{p} \\
& \lesssim\left\|\left[\sum_{j \in \mathbb{N}_{0}^{d}}\left(\prod_{i: \ell_{i}<0} 2^{\ell_{i}(m-s) \theta}\right)\left(\prod_{i: \ell_{i} \geq 0} 2^{-s \ell_{i} \theta}\right) 2^{|j+\ell|_{1} s \theta}\left|P_{2^{j+\ell}, a} f_{j+\ell}\right|^{\theta}\right]^{1 / \theta}\right\|_{p},
\end{aligned}
$$

with $a>\max \{1 / p, 1 / \theta\}$. This is because $i \in e_{0}(j)$ implies $\ell_{i} \geq 0$. Then Theorem 2.4 yields

$$
F_{\ell} \lesssim\left(\prod_{i: \ell_{i}<0} 2^{\ell_{i}(m-s)}\right)\left(\prod_{i: \ell_{i} \geq 0} 2^{-s \ell_{i}}\right)\|f\|_{\mathbf{F}_{p, \theta}^{s}} .
$$

Step 3 In a view of (4.16), we conclude that

$$
\left\|\left(\sum_{j \in \mathbb{N}_{0}^{d}} 2^{|j|_{1} s \theta} \mathcal{R}_{m}^{e(j)}\left(T_{d}^{\psi} f, 2^{-j}, \cdot\right)^{\theta}\right)^{1 / \theta}\right\|_{p} \lesssim\|f\|_{\mathbf{F}_{p, \theta}^{s}} .
$$

The proof is complete.

Proof of Theorem 4.1. Step 1 Let $\left\{\eta_{j}\right\}_{j \in \mathbb{N}_{0}^{d}}$ and $\left\{f_{j}\right\}_{j \in \mathbb{Z}^{d}}$ be defined as in the proof of Theorem 4.2. Analogously to (4.16), we have

$$
\begin{aligned}
\left\|T_{d}^{\psi} f\right\|_{\mathbf{B}_{p, \theta}^{s}}^{(m)} & \leq\left(\sum_{j \in \mathbb{N}_{0}^{d}} 2^{|j|_{1} s \theta}\left(\sum_{\ell \in \mathbb{Z}^{d}}\left\|\mathcal{R}_{m}^{e(j)}\left(T_{d}^{\psi} f_{j+\ell}, 2^{-j}, \cdot\right)\right\|_{p}\right)^{\theta}\right)^{1 / \theta} \\
& \leq\left(\sum_{\ell \in \mathbb{Z}^{d}} \sum_{j \in \mathbb{N}_{0}^{d}} 2^{|j|_{1} s \theta}\left\|\mathcal{R}_{m}^{e(j)}\left(T_{d}^{\psi} f_{j+\ell}, 2^{-j}, \cdot\right)\right\|_{p}^{\theta}\right)^{1 / \theta} .
\end{aligned}
$$


Here we assume $\theta<1$. If $\theta \geq 1$, we use the triangle inequality.

Step 2 The case $p>1$. We put $m=\lfloor s\rfloor+1$. The proof is similar in $\mathbf{F}$-spaces but less technical. Here we use the classical Hardy-Littlewood maximal inequality (the scalar version of Theorem 2.1) and the scalar version of Theorem 2.4. These inequalities do not depend on the parameter $\theta$. Hence the result in the $\mathbf{B}$-spaces can be extended to $0<\theta \leq \infty$.

Step 3 The case $p=1$. Using the convolution representation of the $m$ th order difference in $e_{2}(j, \ell)$, we obtain from (4.19),

$$
\begin{aligned}
& \Delta_{h}^{m, e(j)}\left(T_{d}^{\psi} f_{j+\ell}, x\right) \\
& =\sum_{k} \Delta_{h}^{m, e_{2}(j, \ell)}\left(T_{d}^{\psi} f_{j+\ell}, x+(k \cdot h)\right) \\
& =\sum_{k} \int_{\mathbb{R}^{\left|e_{2}(j, \ell)\right|}} D^{\left(m_{e_{2}(j, \ell)}\right)}\left(T_{d}^{\psi} f_{j+\ell}\right)(x+y+(k \cdot h)) \prod_{i \in e_{2}(j, \ell)} h_{i}^{m-1} B_{m}\left(h_{i}^{-1} y_{i}\right) \mathrm{d} y_{i} .
\end{aligned}
$$

Here $y=\left(y_{1}, \ldots, y_{d}\right)$ with $y_{i}=0$ if $i \notin e_{2}(j, \ell)$. We used the same notation in the proof of Theorem 4.2, see (4.17) and (4.18). Inserting this into $\left\|\mathcal{R}_{m}^{e(j)}\left(T_{d}^{\psi} f, 2^{-j}, \cdot\right)\right\|_{1}$ and then changing the order of integration with the fact that

$$
\prod_{i \in e_{2}(j, \ell)} \int_{\mathbb{R}} h_{i}^{-1} B_{m}\left(h_{i}^{-1} y_{i}\right) \mathrm{d} y_{i}=1,
$$

we obtain

$$
\begin{aligned}
& \left\|\mathcal{R}_{m}^{e(j)}\left(T_{d}^{\psi} f, 2^{-j}, \cdot\right)\right\|_{1} \\
& \quad \lesssim\left(\prod_{i \in e_{2}(j, \ell)} 2^{-j_{i} m}\right) \int_{\Gamma_{j}^{e(j)}}\left\|D^{\left(m_{e_{2}(j, \ell)}\right)}\left(T_{d}^{\psi} f_{j+\ell}\right)(\cdot)\right\|_{1} \prod_{i \in e(j)} 2^{j} \mathrm{~d} h_{i} \\
& \quad \lesssim\left(\prod_{i \in e_{2}(j, \ell)} 2^{-j_{i} m}\right)\left\|D^{\left(m_{e_{2}(j, \ell)}\right)}\left(T_{d}^{\psi} f_{j+\ell}\right)(\cdot)\right\|_{1} \cdot
\end{aligned}
$$

The next step is carried out as F-spaces. Note that in this case, we choose $m=\lfloor s\rfloor+2$, since there must exist $a$ such that $1<a \leq m$ for the inequality (4.22) to hold. The proof is complete.

Remark 4.7 The last step of the proof for $p=1$ shows that, based on our method, we have to guarantee that $\max \{1, s\}<m$. That's why we need the more restrictive condition $k>m+1=\lfloor s\rfloor+2$ in Theorem 1.1. Under the additional assumption $s \geq 1$, we can relax this condition to $k>\lfloor s\rfloor+1$ as in the case $p>1$.

Another possibility to relax this condition is to use the Nikol'skij inequality at the appropriate point, as was already done in [8]. 


\section{Pointwise Multiplication}

We will first comment on the result for $\mathbf{F}$-spaces in Theorem 1.4. Again, we make use of the useful characterization by differences in Theorem 3.7. The technique used in the proof might not be "optimal" in the sense of Remarks 5.2, 5.3 below. However, it is quite transparent and works well for spaces with dominating mixed smoothness $\mathbf{A}_{p, \theta}^{s}$ where almost nothing is known in this direction. Recall that the multiplication operator $\widetilde{T}_{d}^{\psi}$ is defined by

$$
\widetilde{T}_{d}^{\psi}: f(x) \mapsto \psi(x) \cdot f(x), \quad f \in L_{1}\left(\mathbb{R}^{d}\right), x \in \mathbb{R}^{d},
$$

for some sufficiently smooth and compactly supported function $\psi$. Similarly to Lemma 4.4, we will prove a one-dimensional version of Theorem 1.4 first.

Lemma 5.1 Let $0<p<\infty, 0<\theta \leq \infty$, and $s>\sigma_{p, \theta}$. Further, let $\psi \in C_{0}^{k}(\Omega)$ with $k \geq\lfloor s\rfloor+1$ for some compact set $\Omega \subset \mathbb{R}$. Then

$$
\left\|\widetilde{T}_{1}^{\psi} f\right\|_{F_{p, \theta}^{s}} \lesssim\|f\|_{F_{p, \theta}^{s}(\mathbb{T})}, \quad f \in F_{p, \theta}^{s}(\mathbb{T})
$$

\section{Remark 5.2 (Small values of $\mathbf{s}$ )}

(i) We believe that the conditions $s>\sigma_{p, \theta}$ and $s>\sigma_{p}$ in Lemma 5.1 and Theorems 1.3, 1.4 are only technical and caused by our proof technique. On the one hand, $s>\sigma_{p, \theta}$ (and $s>\sigma_{p}$ in the $B$-case) is necessary for the characterization of Triebel-Lizorkin spaces by means of differences, see Theorems 3.6, 3.7 and [4], and on the other hand, our proof below requires this condition. One may relax (or even remove) this condition by using the characterization of Triebel-Lizorkin spaces via local means, see Proposition 3.8 above, [41, Thm. 4.2.2] and [50, Thm. 1.25]. Note that removing the condition $s>\sigma_{p, \theta}$ will extend the range of parameters for which (1.10) holds. In fact, if $1 / \theta-1>1 / p$, then the case $1 / p \leq s<$ $1 / \theta-1$ for the $\mathbf{F}$-scale in (1.10) is not covered by Theorem 1.4 , although it is reasonable since $\mathbf{F}_{p, \theta}^{s} \hookrightarrow C\left(\mathbb{R}^{d}\right)$. In other words, we would obtain that

$$
\operatorname{Int}_{n}\left(\AA_{p, \theta}^{s}\right) \asymp \operatorname{Int}_{n}\left(\mathbf{A}_{p, \theta}^{s}\left(\mathbb{T}^{d}\right)\right), \quad n \in \mathbb{N},
$$

holds true whenever $\mathbf{A}_{p, \theta}^{s}$ is embedded into $C\left(\mathbb{R}^{d}\right)$ which represents a certain minimal condition such that (1.1) makes sense.

(ii) The condition $s>\sigma_{p}$ ensures that the spaces $\mathbf{A}_{p, \theta}^{s}$ consist of regular distributions represented by a locally integrable function, see Lemma 3.5(i) above. Therefore, the pointwise multiplication is well defined. However, inspecting the proofs below, we only need to give sense to the pointwise multiplication for smooth building blocks, see (4.15) above, such that we may define

$$
\widetilde{T}_{d}^{\psi} f:=\sum_{j \in \mathbb{N}_{0}^{d}} \psi(x) f_{j}(x),
$$


where convergence is considered in $\mathcal{S}^{\prime}\left(\mathbb{R}^{d}\right)$. From that point of view, one may even drop the condition $s>\sigma_{p}$ at the price of $k=k(p, s)$ in both $B$ and $F$-spaces. We leave the details to the interested reader.

\section{Remark 5.3 (Pointwise multiplier spaces and optimality)}

(i) We ask for a sharp description of the space of pointwise multipliers for spaces $M\left(\mathbf{A}_{p, \theta}^{s}\left(\mathbb{R}^{d}\right)\right)$. This space might be much larger than $C_{0}^{k}(\Omega)$, which we considered in Theorem 1.4. Questions of this type have some history, see [28, Chapt. 4] and the references therein. In that sense, the result in Lemma 5.1 is far from being optimal. In fact, combining the technique from [28, Thm. 4.9.1] with [42, Thms. $1.26,1.29$ ], one can prove the following rather sharp result: If $A_{p, \theta}^{s}\left(\mathbb{R}^{d}\right.$ ) (which is the isotropic version of (3.1)) embeds into $C\left(\mathbb{R}^{d}\right)$, then $\psi$ is a pointwise multiplier in the sense above if $\psi \in \AA_{p, \theta}^{s}(\Omega)$. With regard to the case $s<n / p$, we refer to [30].

(ii) In the recent preprint [24], by employing the characterization by differences of function spaces with dominating mixed smoothness, Sickel and the first author were able to describe the spaces of all pointwise multipliers for $\mathbf{B}_{p, p}^{s}\left(\mathbb{R}^{d}\right)$ and $\mathbf{W}_{p}^{s}\left(\mathbb{R}^{d}\right)$ under certain conditions. The technique of using paraproducts already applied in [28, Chapt. 4] has not yet been developed for spaces with dominating mixed smoothness $\mathbf{A}_{p, \theta}^{s}$. This seems to be a difficult task and is not straightforward.

\subsection{Proof of Lemma 5.1}

For the proof we need the following variant of [47, Lemma 3.3.1].

Lemma 5.4 Let $a>0, b \geq 1, m \in \mathbb{N}, h \in \mathbb{R} \backslash\{0\}, \psi \in C_{0}^{k}(\mathbb{R})$ with $k \geq m$ and $f \in \mathcal{S}^{\prime}(\mathbb{R})$ such that $\operatorname{supp}(\mathcal{F} f) \subset[-b, b]$. Then it holds for any $t \in \mathbb{R}$ that

$$
\left|\Delta_{h}^{m}(\psi f, t)\right| \leq C_{m, a, \psi} \max \left\{1,|b h|^{a}\right\} \min \left\{1,|b h|^{m}\right\} P_{b, a} f(t) .
$$

Proof Assume first that $b=1$. By the mean value theorem from calculus, we get

$$
\begin{aligned}
\left|\Delta_{h}^{m}(\psi f, t)\right| & \leq|h|^{m} \max \left\{1,|m h|^{a}\right\} \sup _{|\xi| \leq m h} \frac{\left|(\psi f)^{(m)}(t-\xi)\right|}{(1+|\xi|)^{a}} \\
& \leq c_{m, a}|h|^{m} \max \left\{1,|h|^{a}\right\} \sup _{\xi \in \mathbb{R}} \frac{\left|\sum_{j=0}^{m}\left(\begin{array}{c}
m \\
j
\end{array}\right) \psi^{(j)}(t-\xi) f^{(m-j)}(t-\xi)\right|}{(1+|\xi|)^{a}}
\end{aligned}
$$

where we used Leibniz's rule in the second estimate. Let us define

$$
c_{m, \psi}:=2^{m} \max _{j=0, \ldots, m}\left\|\psi^{(j)}\right\|_{\infty}
$$

and continue with the triangle inequality and (2.3) to obtain

$$
\left|\Delta_{h}^{m}(\psi f)\right| \leq c_{m, a} \cdot c_{m, \psi} \cdot|h|^{m} \max \left\{1,|h|^{a}\right\} \cdot \sup _{\xi \in \mathbb{R}} \frac{|f(t-\xi)|}{(1+|\xi|)^{a}} \quad, \quad x \in \mathbb{R} .
$$


What follows is a simple homogeneity argument to deal with $b>1$ and $\operatorname{supp}(\mathcal{F} f) \subset$ $[-b, b]$. Putting $\psi_{b}:=\psi(\cdot / b)$ and $f_{b}:=f(\cdot / b)$, we get $\Delta_{h}^{m}(\psi f)=\Delta_{b h}^{m}\left(\psi_{b} f_{b}\right)(b t)$. Further, we have $c_{m, \psi_{b}} \lesssim c_{m, \psi}$ in (5.2). Due to $\operatorname{supp}\left(\mathcal{F} f_{b}\right) \subset[-1,1]$, we can apply (5.3) to obtain for $t \in \mathbb{R}$ :

$$
\begin{aligned}
\left|\Delta_{h}^{m}(\psi f, t)\right|=\left|\Delta_{b h}^{m}\left(\psi_{b} f_{b}, b t\right)\right| & \leq c_{m, a} \cdot c_{m, \psi} \cdot|b h|^{m} \max \left\{1,|b h|^{a}\right\} \cdot \sup _{\xi \in \mathbb{R}} \frac{\left|f_{b}(b t-\xi)\right|}{(1+|\xi|)^{a}} \\
& =c_{m, a} \cdot c_{m, \psi} \cdot|b h|^{m} \max \left\{1,|b h|^{a}\right\} \cdot \sup _{\xi \in \mathbb{R}} \frac{|f(t-\xi)|}{(1+|b \xi|)^{a}} \\
& =C_{m, a, \psi}|b h|^{m} \max \left\{1,|b h|^{a}\right\} P_{b, a} f(t) .
\end{aligned}
$$

On the other hand, by (3.2), we observe for $t \in \mathbb{R}$ :

$$
\begin{aligned}
\left|\Delta_{h}^{m}(\psi f, t)\right| & \leq \sum_{j=0}^{m}\left|\left(\begin{array}{c}
m \\
j
\end{array}\right) \psi(t+j h)\right| \cdot|f(t+j h)| \\
& \leq c_{m, \psi} c_{m, a} \max \left\{1,|b h|^{a}\right\} \sup _{|\xi| \leq m h} \frac{|f(t-\xi)|}{(1+|b \xi|)^{a}} \\
& \leq C_{m, a, \psi} \max \left\{1,|b h|^{a}\right\} P_{b, a} f(t) .
\end{aligned}
$$

Now, (5.4) together with (5.5) imply (5.1).

Proof of Lemma 5.1. In the case $\min \{p, \theta\} \leq 1$, we choose $0<\lambda<\min \{p, \theta\}$ and $a>1 / \min \{p, \theta\}$ such that $s-(1-\lambda) a>0$. It is possible since $s>\sigma_{p, \theta}$. If $\min \{p, \theta\}>1$, we put $\lambda=1$. Let $\Omega^{\prime}=\Omega+[-m, m]$, and note that $\operatorname{supp}\left(\widetilde{T}_{1}^{\psi} f\right) \subset$ $\operatorname{supp}(\psi) \subset \Omega$ by assumption. Then, clearly with $m \geq\lfloor s\rfloor+1$, we have

$$
\begin{aligned}
\left\|\widetilde{T}_{1}^{\psi} f\right\|_{F_{p, \theta}^{s}}^{(m)} & \lesssim\left\|\left(\sum_{j \in \mathbb{N}_{0}} 2^{j s \theta}\left[2^{j} \int_{-2^{-j}}^{2^{-j}}\left|\Delta_{h}^{m}\left(\widetilde{T}_{1}^{\psi} f, \cdot\right)\right| \mathrm{d} h\right]^{\theta}\right)^{1 / \theta}\right\|_{L_{p}\left(\Omega^{\prime}\right)} \\
& \leq\left\|\left(\sum_{j \in \mathbb{N}_{0}} 2^{j s \theta}\left[\sum_{\ell \in \mathbb{Z}} 2^{j} \int_{-2^{-j}}^{2^{-j}}\left|\Delta_{h}^{m}\left(\widetilde{T}_{1}^{\psi} f_{j+\ell}, \cdot\right)\right| \mathrm{d} h\right]^{\theta}\right)^{1 / \theta}\right\|_{L_{p}\left(\Omega^{\prime}\right)} \\
& \leq\left\|\left(\sum_{\ell<0}\left(\sum_{j \in \mathbb{N}_{0}} 2^{j s \theta}\left[2^{j} \int_{-2^{-j}}^{2^{-j}}\left|\Delta_{h}^{m}\left(\widetilde{T}_{1}^{\psi} f_{j+\ell}, \cdot\right)\right| \mathrm{d} h\right]^{\theta}\right)^{u / \theta}\right)^{1 / u}\right\|_{L_{p}\left(\Omega^{\prime}\right)} \\
& +\left\|\left(\sum_{\ell \geq 0}\left(\sum_{j \in \mathbb{N}_{0}} 2^{j s \theta}\left[2^{j} \int_{-2^{-j}}^{2^{-j}}\left|\Delta_{h}^{m}\left(\widetilde{T}_{1}^{\psi} f_{j+\ell}, \cdot\right)\right| \mathrm{d} h\right]^{\theta}\right)^{u / \theta}\right)^{1 / u}\right\|_{L_{p}\left(\Omega^{\prime}\right)},
\end{aligned}
$$

where $u=\min \{1, \theta\}$. By (5.1), we obtain for $\ell<0$ that

$$
2^{j} \int_{-2^{-j}}^{2^{-j}}\left|\Delta_{h}^{m}\left(\widetilde{T}_{1}^{\psi} f_{j+\ell}, \cdot\right)\right| \mathrm{d} h \lesssim 2^{\ell m} P_{2^{\ell+j}, a} f_{j+\ell}(t),
$$


and hence

$$
\begin{gathered}
\left\|\left(\sum_{\ell<0}\left(\sum_{j \in \mathbb{N}_{0}} 2^{j s \theta}\left[2^{j} \int_{-2^{-j}}^{2^{-j}}\left|\Delta_{h}^{m}\left(\widetilde{T}_{1}^{\psi} f_{j+\ell}, \cdot\right)\right| \mathrm{d} h\right]^{\theta}\right)^{u / \theta}\right)^{1 / u}\right\|_{L_{p}\left(\Omega^{\prime}\right)} \\
\leq\left\|\left(\sum_{\ell<0} 2^{\ell(m-s) u}\left(\sum_{j \in \mathbb{N}_{0}} 2^{(j+\ell) s \theta}\left|P_{2^{j+\ell}, a} f_{j+\ell}\right|^{\theta}\right)^{u / \theta}\right)^{1 / u}\right\|_{L_{p}\left(\Omega^{\prime}\right)} \\
\lesssim\left\|\left(\sum_{j \in \mathbb{N}_{0}} 2^{j s \theta}\left|P_{2^{j}, a} f_{j}\right|^{\theta}\right)^{1 / \theta}\right\|_{L_{p}(\mathbb{T})},
\end{gathered}
$$

since the function inside the $L_{p}$-(quasi-)norm is 1-periodic. Applying the periodic version of Theorem 2.4 with $a>\max \{1 / p, 1 / \theta\}$, see [29, Thm. 3.4.1], we obtain

$$
\left\|\left(\sum_{\ell<0}\left(\sum_{j \in \mathbb{N}_{0}} 2^{j s \theta}\left[2^{j} \int_{-2^{-j}}^{2^{-j}}\left|\Delta_{h}^{m}\left(\widetilde{T}_{1}^{\psi} f_{j+\ell}, \cdot\right)\right| \mathrm{d} h\right]^{\theta}\right)^{u / \theta}\right)^{1 / u}\right\|_{L_{p}\left(\Omega^{\prime}\right)} \lesssim\|f\|_{F_{p, \theta}^{s}(\mathbb{T})}
$$

Let us deal with $\ell \geq 0$. We write

$$
\left|\Delta_{h}^{m}\left(\psi f_{j+\ell}, t\right)\right|=\left|\Delta_{h}^{m}\left(\psi f_{j+\ell}, t\right)\right|^{1-\lambda} \cdot\left|\Delta_{h}^{m}\left(\psi f_{j+\ell}, t\right)\right|^{\lambda}
$$

By (5.1), we obtain

$$
\left|\Delta_{h}^{m}\left(\psi f_{j+\ell}, t\right)\right|^{1-\lambda} \lesssim 2^{\ell a(1-\lambda)}\left|P_{2^{j+\ell}, a} f_{j+\ell}(t)\right|^{1-\lambda}
$$

This gives

$$
2^{j} \int_{-2^{-j}}^{2^{-j}}\left|\Delta_{h}^{m}\left(\psi f_{j+\ell}, t\right)\right|^{\lambda} \mathrm{d} h \leq M\left[\left|f_{j+\ell}\right|^{\lambda}\right](t)
$$

and therefore

$$
\begin{aligned}
& \sum_{\ell \geq 0}\left(\sum_{j \in \mathbb{N}_{0}} 2^{j s \theta}\left[2^{j} \int_{-2^{-j}}^{2^{-j}}\left|\Delta_{h}^{m}\left(\widetilde{T}_{1}^{\psi} f_{j+\ell}, \cdot\right)\right| \mathrm{d} h\right]^{\theta}\right)^{u / \theta} \\
& \quad \lesssim \sum_{\ell \geq 0}\left(\sum_{j \in \mathbb{N}_{0}} 2^{j s \theta} 2^{\ell a(1-\lambda) \theta}\left[\left|P_{2^{j+\ell}, a} f_{j+\ell}(t)\right|^{1-\lambda} M\left[\left|f_{j+\ell}\right|^{\lambda}\right](t)\right]^{\theta}\right)^{u / \theta} \\
& \quad \lesssim \sum_{\ell \geq 0} 2^{[a(1-\lambda)-s] \ell u}\left(\sum_{j \in \mathbb{N}_{0}} 2^{(j+\ell) s \theta}\left[\left|P_{2^{j+\ell}, a} f_{j+\ell}(t)\right|^{1-\lambda} M\left[\left|f_{j+\ell}\right|^{\lambda}\right](t)\right]^{\theta}\right)^{u / \theta} \\
& \lesssim\left(\sum_{j \in \mathbb{N}_{0}} 2^{j s \theta}\left[\left|P_{2^{j}, a} f_{j}(t)\right|^{1-\lambda} M\left[\left|f_{j}\right|^{\lambda}\right](t)\right]^{\theta}\right)^{u / \theta} .
\end{aligned}
$$


Plugging this into (5.6) and using Hölder's inequality twice with $1 / \lambda$ and $1 /(1-\lambda)$ gives

$$
\begin{aligned}
& \left\|\left(\sum_{\ell \geq 0}\left(\sum_{j \in \mathbb{N}_{0}} 2^{j s \theta}\left[2^{j} \int_{-2^{-j}}^{2^{-j}}\left|\Delta_{h}^{m}\left(\widetilde{T}_{1}^{\psi} f_{j+\ell}, \cdot\right)\right| \mathrm{d} h\right]^{\theta}\right)^{u / \theta}\right)^{1 / u}\right\|_{L_{p}\left(\Omega^{\prime}\right)} \\
& \lesssim\left\|\left(\sum_{j \in \mathbb{N}_{0}} 2^{j s \theta}\left|P_{2^{j}, a} f_{j}\right|^{\theta}\right)^{1 / \theta}\right\|_{L_{p}(\mathbb{T})}^{1-\lambda} \cdot\left\|\left(\sum_{j \in \mathbb{N}_{0}}\left[2^{j s \lambda} M\left(\left|P_{2^{j}, a} f_{j}\right|^{\lambda}\right)\right]^{\frac{\theta}{\lambda}}\right)^{\frac{\lambda}{\theta}}\right\|_{L_{\frac{p}{\lambda}}(\mathbb{T})}
\end{aligned}
$$

Applying the periodic versions of Theorems 2.4 and 2.1 (note that $\lambda<\min \{p, \theta\}$ ), see [29, Prop. 3.2.4, Thm. 3.4.1], we get

$$
\left\|\left(\sum_{\ell \geq 0}\left(\sum_{j \in \mathbb{N}_{0}} 2^{j s \theta}\left[2^{j} \int_{-2^{-j}}^{2^{-j}}\left|\Delta_{h}^{m}\left(\widetilde{T}_{1}^{\psi} f_{j+\ell}, \cdot\right)\right| \mathrm{d} h\right]^{\theta}\right)^{u / \theta}\right)^{1 / u}\right\|_{L_{p}\left(\Omega^{\prime}\right)} \lesssim\|f\|_{F_{p, \theta}^{s}(\mathbb{T})} .
$$

From (5.7) and (5.8), we obtain the desired estimate. The proof is complete.

\subsection{Proof of Theorems 1.3 and 1.4}

In order to prove Theorems 1.3 and 1.4, we need a multivariate version of Lemma 5.4.

Lemma 5.5 Let $b=\left(b_{1}, \ldots, b_{d}\right)>0, a>0, e \subset[d], m \in \mathbb{N}, \psi \in C_{0}^{k}\left(\mathbb{R}^{d}\right)$ with $k \geq m$ and $h=\left(h_{1}, \ldots, h_{d}\right) \in \mathbb{R}^{d}$. Further, let $f \in \mathcal{S}^{\prime}\left(\mathbb{R}^{d}\right)$ with $\operatorname{supp}(\mathcal{F} f) \subset Q_{b}$, where

$$
Q_{b}:=\left[-b_{1}, b_{1}\right] \times \cdots \times\left[-b_{d}, b_{d}\right] .
$$

Then there exists a constant $C>0$ (independent of $f, b$, and $h$ ) such that

$$
\left|\Delta_{h}^{m, e}(\psi f, x)\right| \leq C_{m, a, \psi} \prod_{i \in e} \max \left\{1,\left|b_{i} h_{i}\right|^{a}\right\} \min \left\{1,\left|b_{i} h_{i}\right|^{m}\right\} \cdot P_{b, a}^{e} f(x)
$$

holds for all $x \in \mathbb{R}^{d}$. Here

$$
P_{b, a}^{e} f(x):=\sup _{y_{i} \in \mathbb{R}, i \in e} \frac{|f(y)|}{\prod_{i \in e}\left(1+\left|b_{i}\left(x_{i}-y_{i}\right)\right|\right)^{a}}
$$

denotes the counterpart of (2.2) for $e \subset[d]$.

Proof The proof goes along the same lines of the proof of Lemma 5.4, see also [47, Lem. 3.3.2].

Now we are in position to prove Theorems 1.3 and 1.4. 
Proof of Theorem 1.4. We will show that with the assumption in Theorem 1.4, we have

$$
\left\|\left(\sum_{j \in \mathbb{N}_{0}^{d}} 2^{s|j|{ }_{1} \theta} \mathcal{R}_{m}^{e(j)}\left(\widetilde{T}_{d}^{\psi} f, 2^{-j}, \cdot\right)^{\theta}\right)^{1 / \theta}\right\|_{L_{p}\left(\mathbb{R}^{d}\right)} \lesssim\|f\|_{\mathbf{F}_{p, \theta}^{s}\left(\mathbb{T}^{d}\right)}
$$

for all $f \in \mathbf{F}_{p, \theta}^{s}\left(\mathbb{T}^{d}\right)$, where $\mathcal{R}_{m}^{e(j)}\left(\psi f, 2^{-j}, \cdot\right)$ is defined in (3.3). Let us first prove the case $\min \{p, \theta\} \leq 1$. We choose $0<\lambda<\min \{p, \theta\}$ and $a>1 / \min \{p, \theta\}$ as in the proof of Lemma 5.1. Using the decomposition of unity, $\operatorname{supp}(\psi) \subset \Omega$, and $\Omega^{\prime}=\Omega+[-m, m]^{d}$, we get

$$
\begin{gathered}
\left\|\left(\sum_{j \in \mathbb{N}_{0}^{d}} 2^{|j|_{1} s \theta} \mathcal{R}_{m}^{e(j)}\left(\widetilde{T}_{d}^{\psi} f, 2^{-j}, \cdot\right)^{\theta}\right)^{1 / \theta}\right\|_{p} \\
\leq\left\|\sum_{\ell \in \mathbb{Z}^{d}}\left(\sum_{j \in \mathbb{N}_{0}^{d}} 2^{|j|_{1} s \theta} \mathcal{R}_{m}^{e(j)}\left(\psi f_{j+\ell}, 2^{-j}, \cdot\right)^{\theta}\right)^{1 / \theta}\right\|_{L_{p}\left(\Omega^{\prime}\right)},
\end{gathered}
$$

see (4.16). For simplicity, we assume that $\theta \geq 1$. Note that $p<1$ is still possible here. The modifications in case $\theta<1$ are straightforward, see the proof of Lemma 5.1. From (3.3), we have for $x \in \mathbb{R}^{d}$,

$$
2^{|j|_{1} s} \mathcal{R}_{m}^{e(j)}\left(\psi f_{j+\ell}, 2^{-j}, x\right) \lesssim 2^{|j|_{1} s} \int_{\Gamma_{j}^{e(j)}}\left|\Delta_{h}^{m, e(j)}\left(\psi f_{j+\ell}, x\right)\right| \prod_{i \in e(j)} 2^{j_{i}} \mathrm{~d} h_{i},
$$

where $\Gamma_{j}^{e(j)}, e_{1}(j, \ell)$ and $e_{2}(j, \ell)$ are defined above in (4.17) and (4.18). Lemma 5.5, see also [48, Lemma 1.12], yields

$$
\begin{aligned}
\mid \Delta_{h}^{m, e(j)} & \left(\psi f_{j+\ell}, x\right)|=| \Delta_{h}^{m, e_{2}(j, \ell)} \circ \Delta_{h}^{m, e_{1}(j, \ell)}\left(\psi f_{j+\ell}, x\right) \mid \\
& \lesssim\left(\prod_{i \in e_{2}(j, \ell)}\left|2^{j_{i}+\ell_{i}} h_{i}\right|^{m}\right)\left(P_{2^{j+\ell}, a}^{e_{2}(j, \ell)} \Delta_{h}^{m, e_{1}(j, \ell)}\left(\psi f_{j+\ell}\right)\right)(x)
\end{aligned}
$$

Applying Lemma 5.5 with the components in $e_{1}(j, \ell)$, we get

$$
\begin{aligned}
& \left(P_{2^{j+\ell}, a}^{e_{2}(j, \ell)} \Delta_{h}^{m, e_{1}(j, \ell)}\left(\psi f_{j+\ell}\right)\right)(x) \\
& =\left|\left(P_{2^{j+\ell}, a}^{e_{2}(j, \ell)} \Delta_{h}^{m, e_{1}(j, \ell)}\left(\psi f_{j+\ell}\right)\right)(x)\right|^{1-\lambda} \cdot\left|\left(P_{2^{j+\ell}, a}^{e_{2}(j, \ell)} \Delta_{h}^{m, e_{1}(j, \ell)}\left(\psi f_{j+\ell}\right)\right)(x)\right|^{\lambda} \\
& \lesssim\left[\left(\prod_{i \in e_{1}(j, \ell)}\left|2^{j_{i}+\ell_{i}} h_{i}\right|^{a}\right) P_{2^{j+\ell}, a}^{e(j)} f_{j+\ell}(x)\right]^{1-\lambda} \cdot\left|\left(P_{2^{j+\ell}, a}^{e_{2}(j, \ell)} \Delta_{h}^{m, e_{1}(j, \ell)}\left(\psi f_{j+\ell}\right)\right)(x)\right|^{\lambda} .
\end{aligned}
$$

Expanding $\Delta_{h}^{m, e_{1}(j, \ell)}\left(\psi f_{j+\ell}, x\right)$, we obtain

$$
\begin{aligned}
\left(P_{2^{j+\ell, a}}^{e_{2}(j, \ell)} \Delta_{h}^{m, e_{1}(j, \ell)}\left(\psi f_{j+\ell}\right)\right)(x) & \lesssim \sum_{u}\left(P_{2^{j+\ell, a}}^{e_{2}(j, \ell)}\left(\psi f_{j+\ell}\right)\right)(x+(u \cdot h)) \\
& \lesssim \sum_{u}\left(P_{2^{j+\ell, a}, e_{j}(j, \ell)}^{e_{j+\ell}}\right)(x+(u \cdot h)) .
\end{aligned}
$$


Here the sum is taken over all $u \in \mathbb{N}_{0}^{d}$ such that $0 \leq u_{i} \leq m$ if $u_{i} \in e_{1}(j, \ell)$ and $u_{i}=0$ if $i \notin e_{1}(j, \ell)$. In the last inequality, we used the definition of the Peetre maximal function. This leads to

$$
\begin{aligned}
\left|\Delta_{h}^{m, e(j)}\left(\psi f_{j+\ell}, x\right)\right| \lesssim & \left(\prod_{i \in e_{2}(j, \ell)} 2^{\ell_{i} m}\right)\left(\prod_{i \in e_{1}(j, \ell)} 2^{\ell_{i} a(1-\lambda)}\right)\left[P_{2^{j+\ell}, a}^{e(j)} f_{j+\ell}(x)\right]^{1-\lambda} \\
& \times \sum_{u}\left[\left(P_{2^{j+\ell}, a}^{e_{2}(j, \ell)} f_{j+\ell}\right)(x+(u \cdot h))\right]^{\lambda} .
\end{aligned}
$$

Inserting this into (5.10) and performing the integration yields

$$
\begin{aligned}
2^{|j|_{1} s} & \mathcal{R}_{m}^{e(j)}\left(\psi f_{j+\ell}, 2^{-j}, x\right) \\
\lesssim & 2^{|j|_{1} s}\left(\prod_{i \in e_{2}(j, \ell)} 2^{\ell_{i} m}\right)\left(\prod_{i \in e_{1}(j, \ell)} 2^{\ell_{i} a(1-\lambda)}\right)\left[P_{2^{j+\ell}, a}^{e(j)} f_{j+\ell}(x)\right]^{1-\lambda} . \\
& \times M_{e_{1}(j, \ell)}\left(\left|P_{2^{j+\ell}, a}^{e_{2}(j, \ell)} f_{j+\ell}(x)\right|^{\lambda}\right) \\
\lesssim & 2^{|j|_{1} s}\left(\prod_{i \in e_{2}(j, \ell)} 2^{\ell_{i} m}\right)\left(\prod_{i \in e_{1}(j, \ell)} 2^{\ell_{i} a(1-\lambda)}\right)\left[P_{2^{j+\ell}, a} f_{j+\ell}(x)\right]^{1-\lambda} \\
& \times M_{[d]}\left(\left|P_{2^{j+\ell}, a} f_{j+\ell}(x)\right|^{\lambda}\right) .
\end{aligned}
$$

Observe that

$$
\begin{aligned}
2^{|j|_{1} s} & \left(\prod_{i \in e_{2}(j, \ell)} 2^{\ell_{i} m}\right)\left(\prod_{i \in e_{1}(j, \ell)} 2^{\ell_{i} a(1-\lambda)}\right) \\
\quad= & 2^{|j+\ell|_{1} s}\left(\prod_{i \in e_{2}(j, \ell)} 2^{\ell_{i}(m-s)}\right)\left(\prod_{i \in e_{1}(j, \ell)} 2^{\ell_{i}[a(1-\lambda)-s]}\right)\left(\prod_{i \in e_{0}(j)} 2^{-\ell_{i} s}\right) \\
\quad \leq & 2^{|j+\ell|_{1} s}\left(\prod_{i \in e_{2}(j, \ell)} 2^{\ell_{i}(m-s)}\right)\left(\prod_{i \in e_{1}(j, \ell) \cup e_{0}(j)} 2^{-\ell_{i} \delta}\right) \\
\quad= & 2^{|j+\ell|_{1} s}\left(\prod_{i: \ell_{i}<0} 2^{\ell_{i}(m-s)}\right)\left(\prod_{i: \ell_{i} \geq 0} 2^{-\ell_{i} \delta}\right),
\end{aligned}
$$

where $\delta=\min \{s-a(1-\lambda), s\}>0$. This allows us to estimate

$$
\begin{aligned}
\sum_{\ell \in \mathbb{Z}^{d}}\left(\sum_{j \in \mathbb{N}_{0}^{d}} 2^{|j|{ }_{1} s \theta}\right. & \left.\mathcal{R}_{m}^{e(j)}\left(\psi f_{j+\ell}, 2^{-j}, \cdot\right)^{\theta}\right)^{1 / \theta} \\
& \lesssim\left(\sum_{j \in \mathbb{N}_{0}^{d}} 2^{|j|{ }_{1} s \theta}\left[\left|P_{2^{j}, a} f_{j}(x)\right|^{1-\lambda} \cdot M_{[d]}\left(\left|P_{2^{j}, a} f_{j}(x)\right|^{\lambda}\right)\right]^{\theta}\right)^{1 / \theta}
\end{aligned}
$$


Applying Hölder's inequality twice with the pair $(1 /(1-\lambda), 1 / \lambda)$ and afterwards using the periodic version of Theorem 2.1, see [47, Thm. 4.1.2] and [48, Prop. 1.3], we arrive at

$$
\begin{aligned}
& \left\|\sum_{\ell \in \mathbb{Z}^{d}}\left(\sum_{j \in \mathbb{N}_{0}^{d}} 2^{|j|_{1} s \theta} \mathcal{R}_{m}^{e(j)}\left(\psi f_{j+\ell}, 2^{-j}, \cdot\right)^{\theta}\right)^{1 / \theta}\right\|_{L_{p}\left(\Omega^{\prime}\right)} \\
& \lesssim\left\|\left(\sum_{j \in \mathbb{N}_{0}^{d}} 2^{|j|_{1} s \theta}\left|P_{2^{j}, a} f_{j}\right|^{\theta}\right)^{1 / \theta}\right\|_{L_{p}\left(\mathbb{T}^{d}\right)}^{1-\lambda} \cdot\left\|\left(\sum_{j \in \mathbb{N}_{0}^{d}}\left[2^{|j|_{1} s \lambda} M_{[d]}\left(\left|P_{2^{j}, a} f_{j}\right|^{\lambda}\right)\right]^{\frac{\theta}{\lambda}}\right)^{\frac{\lambda}{\theta}}\right\|_{L_{\frac{p}{\lambda}}\left(\mathbb{T}^{d}\right)} \\
& \lesssim\left\|\left(\sum_{j \in \mathbb{N}_{0}^{d}} 2^{|j|_{1} s \theta}\left|P_{2^{j}, a} f_{j}\right|^{\theta}\right)^{1 / \theta}\right\|_{L_{p}\left(\mathbb{T}^{d}\right)},
\end{aligned}
$$

since the function inside the $L_{p}$-(quasi-)norm is 1-periodic. The periodic version of Theorem 2.4, see [47, Thm. 4.1.3] and [48, Prop. 1.5], implies the inequality (5.9). The case $\min \{p, \theta\}>1$ is less technical. We choose $\lambda=1$. Next we take the integral in (5.10) and then estimate above by the Hardy-Littlewood maximal function according to the directions in $e_{1}(j, \ell)$. The rest is carried out similarly to the case $\min \{p, \theta\} \leq 1$. The proof is complete.

Remark 5.6 The proof of Theorem 1.3 (B-case) is similar but technically less involved. As in the F-case, we choose $0<\lambda<p$ and $a>1 / p$ such that $s-a(1-\lambda)>0$ if $p \leq 1$; otherwise we put $\lambda=1$. Again, one can do so because of $s>\sigma_{p}$. Note, that the weaker condition $s>\sigma_{p}$ comes from the fact that the Peetre maximal function (2.2) is bounded in $L_{p}\left(\mathbb{T}^{d}\right), 0<p<\infty$, if $a>1 / p$, which is the scalar version of Theorem 2.4.

Acknowledgements Open access funding provided by Johannes Kepler University Linz. The authors acknowledge the fruitful discussions with D. Bazarkhanov, W. Sickel, V.N. Temlyakov, H. Triebel, and Dinh Dũng on this topic, especially at the ICERM Semester Programme "High-Dimensional Approximation" in Providence, 2014, where this project was initiated, and at the conference "Approximation Methods and Function Spaces" in Hasenwinkel, 2015. Moreover, Van Kien Nguyen would like to thank the Ministry of Education and Training of Vietnam for financial support. Tino Ullrich gratefully acknowledges support by the German Research Foundation (DFG) Ul-403/2-1 and the Emmy-Noether Programme, Ul-403/1-1.

Open Access This article is distributed under the terms of the Creative Commons Attribution 4.0 International License (http://creativecommons.org/licenses/by/4.0/), which permits unrestricted use, distribution, and reproduction in any medium, provided you give appropriate credit to the original author(s) and the source, provide a link to the Creative Commons license, and indicate if changes were made.

\section{References}

1. Amanov, T.I.: Spaces of Differentiable Functions with Dominating Mixed Derivatives. Nauka Kaz. SSR, Alma-Ata (1976)

2. Bakhvalov, N.S.: Optimal convergence bounds for quadrature processes and integration methods of Monte Carlo type for classes of functions. Zh. Vychisl. Mat. i Mat. Fiz. 4, 5-63 (1963)

3. Bykovskii, V.A.: On the correct order of the error of optimal cubature formulas in spaces with dominant derivative, and on quadratic deviations of grids. Akad. Sci. USSR, Vladivostok, Computing Center Far-Eastern Scientific Center (Preprint, 1985) 
4. Christ, M., Seeger, A.: Necessary conditions for vector-valued operator inequalities in harmonic analysis. Proc. Lond. Math. Soc. (3) 93(2), 447-473 (2006)

5. DeVore, R.A., Lorentz, G.G.: Constructive Approximation. Springer, New York (1993)

6. Dick, J., Pillichshammer, F.: Discrepancy theory and quasi-Monte Carlo integration. In: A panorama of discrepancy theory. Lecture Notes in Math, vol. 2107, pp. 539-619. Springer, Cham (2014)

7. Dubinin, V.V.: Cubature formulas for classes of functions with bounded mixed difference. Matem. Sbornik 183(7), (1992) (Math. USSR Sbornik )76(283-292), (1993)

8. Dubinin, V.V.: Cubature formulae for Besov classes. Izv. Math. 61, 259-283 (1997)

9. Dũng, D., Temlyakov, V.N., Ullrich, T.: Hyperbolic cross approximation. Advanced Courses in Mathematics. CRM Barcelona. Birkhäuser/Springer, to appear. arXiv:1601.03978v2 [math.NA]

10. Dũng, D., Ullrich, T.: Lower bounds for the integration error for multivariate functions with mixed smoothness and optimal Fibonacci cubature for functions on the square. Math. Nachr. 288, 743-762 (2015)

11. Fefferman, C., Stein, E.M.: Some maximal inequalities. Am. J. Math. 93, 107-115 (1971)

12. Frolov, K.K.: Upper error bounds for quadrature formulas on function classes. Dokl. Akad. Nauk SSSR 231, 818-821 (1976)

13. Glasserman, P.: Monte Carlo Methods in Financial Engineering. Applications of Mathematics : Stochastic Modelling and Applied Probability. Springer, Berlin (2004)

14. Goda, T., Suzuki, K., Yoshiki, T.: Optimal order quasi-Monte Carlo integration in weighted Sobolev spaces of arbitrary smoothness. IMA J. Numer. Anal. 37, 505-518 (2017)

15. Goda, T., Suzuki, K., Yoshiki, T.: An explicit construction of optimal order quasi-Monte Carlo rules for smooth integrands. SIAM J. Numer. Anal. 54, 2664-2683 (2016)

16. Hinrichs, A.: Discrepancy of Hammersley points in Besov spaces of dominating mixed smoothness. Math. Nachr. 283(3), 478-488 (2010)

17. Hinrichs, A., Markhasin, L., Oettershagen, J., Ullrich, T.: Optimal quasi-Monte Carlo rules on order 2 digital nets for the numerical integration of multivariate periodic functions. Numer. Math. 134, 163-196 (2016)

18. Hlawka, E.: Zur angenäherten Berechnung mehrfacher integrale. Monatsh. Math. 66, 140-151 (1962)

19. Kacwin, C., Oettershagen, J., Ullrich, T.: On the orthogonality of the Chebyshev-Frolov lattice and applications. ArXiv e-prints (2016) arXiv:1606.00492 [math.NA]

20. Korobov, N.M.: Approximate evaluation of repeated integrals. Dokl. Akad. Nauk SSSR 124, 12071210 (1959)

21. Krieg, D., Novak, E.: A universal algorithm for multivariate integration. Found. Comput. Math. (2016). doi:10.1007/s10208-016-9307-y

22. Kuo, F., Sloan, I.H., Woźniakowski, H.: Periodization strategy may fail in high dimensions. Numer. Algorithms 46, 369-391 (2007)

23. Markhasin, L.: Discrepancy and integration in function spaces with dominating mixed smoothness. Diss. Math. 494, 1-81 (2013)

24. Nguyen, V.K., Sickel, W.: Pointwise multipliers for Sobolev and Besov spaces of dominating mixed smoothness. J. Math. Anal. Appl. (2017). doi:10.1016/j.jmaa.2017.02.046

25. Nikol'skij, S.M.: Approximation of Functions of Several Variables and Embedding Theorems. Nauka Moskva, Moscow (1977)

26. Novak, E., Woźniakowski, H.: Tractability of Multivariate Problems: Standard Information for Functionals, vol. II. EMS Tracts in Mathematics, 12. European Mathematical Society (EMS), Zürich, (2010)

27. Nuyens, D., Cools, R.: Higher order quasi Monte Carlo methods: a comparison. AIP Conf. Proc. 1281, 553-557 (2010)

28. Runst, T., Sickel, W.: Sobolev Spaces of Fractional Order, Nemytskij Operators, and Nonlinear Partial Differential Equations. de Gruyter Series in Nonlinear Analysis and Applications, vol. 3. Walter de Gruyter \& Co, Berlin (1996)

29. Schmeisser, H.-J., Triebel, H.: Topics in Fourier Analysis and Function Spaces. Geest \& Portig, Leipzig (1987) and Wiley, Chichester (1987)

30. Sickel, W.: On pointwise multipliers for $F_{p, q}^{s}$. The case $\sigma_{p, q}<s<n / p$. Annali di Matematica pura ed applicata 174, 209-250 (1999)

31. Skriganov, M.M.: Constructions of uniform distributions in terms of geometry of numbers. Algebra $\mathrm{i}$ Analiz 6, 200-230 (1994)

32. Stöckert, B.: Ungleichungen vom Plancherel-Polya-Nikolskij-Typ in gewichteten $L_{p}^{\Omega}$-Räumen mit gemischten Normen. Math. Nachr. 86, 19-32 (1978) 
33. Temlyakov, V.N.: On reconstruction of multivariate periodic functions based on their values at the knots of number-theoretical nets. Anal. Math. 12, 287-305 (1986)

34. Temlyakov, V.N.: On a way of obtaining lower estimates for the errors of quadrature formulas. Mat. Sb. 181(10), 1403-1413 (1990)

35. Temlyakov, V.N.: Error estimates for Fibonacci quadrature formulas for classes of functions with a bounded mixed derivative. Trudy Mat. Inst. Steklov. 200, 327-335 (1991)

36. Temlyakov, V.N.: Approximation of Periodic Functions, Computational Mathematics and Analysis Series. Nova Science Publishers Inc, Commack, NY (1993)

37. Temlyakov, V.N.: On error estimates for cubature formulas. Trudy Mat. Inst. Steklov 207, 326-338 (1994); Proc. Steklov Inst. Math. 207(6), 299-302 (1995)

38. Temlyakov, V.N.: Cubature formulas, discrepancy, and nonlinear approximation. J. Complex. 19, 352391 (2003)

39. Temlyakov, V.: Constructive sparse trigonometric approximation and other problems for functions with mixed smoothness. Mat. Sb. 206, 131-160 (2015)

40. Triebel, H.: Theory of Function Spaces. Birkhäuser, Basel (1983)

41. Triebel, H.: Theory of Function Spaces II. Birkhäuser, Basel (1992)

42. Triebel, H.: Function Spaces and Wavelets on Domains. EMS Publ. House, Zürich (2008)

43. Triebel, H.: Bases in Function Spaces, Sampling Discrepancy, Numerical Integration. EMS Tracts in Mathematics. European Mathematical Society (EMS), Zürich (2010)

44. Ullrich, M.: On "Upper error bounds for quadrature formulas on function classes" by Frolov, K. K. In: Cools, R., Nuyens, D. (eds.) Monte Carlo and Quasi-Monte Carlo Methods, Proceedings in Mathematics \& Statistics, vol. 163, pp. 571-582. Springer, Cham (2016)

45. Ullrich, M.: A Monte Carlo method for integration of multivariate smooth functions. SIAM J. Numer. Anal. (to appear)

46. Ullrich, M., Ullrich, T.: The role of Frolov's cubature formula for functions with bounded mixed derivative. SIAM J. Numer. Anal. 54, 969-993 (2016)

47. Ullrich, T.: Function spaces with dominating mixed smoothness; characterization by differences. Jenaer Schriften zur Mathematik und Informatik, Math/Inf/05/06 (2006)

48. Ullrich, T.: Smolyaks algorithm, sparse grid approximation and periodic function spaces with dominating mixed smoothness. Ph.D Thesis, Friedrich-Schiller-Universität Jena, Jena (2007)

49. Ullrich, T.: Local mean characterization of Besov-Triebel-Lizorkin type spaces with dominating mixed smoothness on rectangular domains. Preprint, Uni Bonn (2008)

50. Vybíral, J.: Function spaces with dominating mixed smoothness. Diss. Math. 436, 73 (2006)

51. Yserentant, H.: Regularity and Approximability of Electronic Wave Functions. In: Lecture Notes in Mathematics. Springer, Berlin (2010) 